\title{
Aerodynamic Shape Optimization of the Common Research Model Wing-Body-Tail Configuration
}

\author{
Song Chen*, Zhoujie Lyu ${ }^{\dagger}$, Gaetan K. W. Kenway ${ }^{\ddagger}$, and Joaquim R. R. A. Martins ${ }^{\S}$ \\ Department of Aerospace Engineering, University of Michigan, Ann Arbor, MI
}

\begin{abstract}
Wing shape is one of the main drivers of aircraft aerodynamic performance, so most aerodynamic shape optimization efforts have been focused solely on the wing. However, the performance of the full aircraft configuration must account for the fact that the aircraft needs to be trimmed. Thus, to realize the full benefit of aerodynamic shape optimization, one should optimize the wing shape while including the full configuration and a trim constraint. To evaluate the benefit of this approach, we perform the aerodynamic shape optimization of the Common Research Model wing-body-tail configuration using gradient-based optimization with a Reynolds-averaged Navier-Stokes (RANS) model that includes a discrete adjoint implementation. We investigate the aerodynamic shape optimization of the wing with a trim constraint that is satisfied by rotating the horizontal tail. We optimize the same wing-body configuration without the tail, but with an added trim drag penalty based on a surrogate model we created. We also conduct the simultaneous aerodynamic shape optimization of both the wing and the horizontal-tail to investigate the benefit of optimizing the tail shape. The design variables consist of 816 wing shape variables and 144 horizontal tail shape variables, as well as the tail rotation angle and the aircraft angle of attack. The drag coefficient is minimized subject to lift and trim constraints. In addition, 1000 geometric thickness constraints and a wing volume constraint are enforced to guarantee that the wing thickness and volume values are no lower than those of the baseline geometry. We found that considering the trim during optimization is a better approach than using a fixed wing moment constraint. We also showed that the trim drag surrogate model we created can be used to yield a design whose drag coefficient is within 1.2 counts of that of an optimization where trim is satisfied with a rotating tail. However, we recommend the simultaneous optimization of wing and tail rotation to obtain the best possible performance.
\end{abstract}

\section{Introduction}

The aerodynamic design of aircraft configurations has benefited tremendously from the development of computational fluid dynamics (CFD) models, which have replaced much of the wind-tunnel testing, and shortened the design cycle by making it easier to try design variations. The use of numerical optimization has the potential to further improve the aerodynamic design process by automating the change in the design and by seeking optimal designs. Since the design of three-dimensional shapes for aerodynamic performance requires a hundreds of design variables, most researchers have resorted to tackling this problem using gradient-based optimizer with adjoint methods for computing the gradients, a technique pioneered by Jameson [1,2]. Given the ever increasing power of high-performance parallel computing, it is now feasible to perform aerodynamic shape optimization based on the Reynolds-averaged Navier-Stokes (RANS) equations on complex geometries with hundreds of design variables [3, 4].

The wing and horizontal-tail play key roles in the aerodynamic performance of conventional aircraft configurations, as they directly affect the lift, drag, and moment of the aircraft. Since the benefit of drag reduction by optimizing the wing alone might not be realized once the full aircraft configuration is trimmed (i.e., when the aircraft is in equilibrium with respect to forces and moments), it is important to consider the trim constraint when performing the optimization. In typical transonic transport aircraft, trim is achieved by rotating the whole horizontal tail. This trim constraint can be enforced without modeling the tail by constraining the moment of the wing alone [3]. While this prevents the

\footnotetext{
*Visiting Scholar, Department of Aerospace Engineering, University of Michigan, AIAA Student Member

${ }^{\dagger} \mathrm{PhD}$ Candidate, Department of Aerospace Engineering, University of Michigan, AIAA Student Member

$¥$ Post Doctoral Research Fellow, Department of Aerospace Engineering, University of Michigan, AIAA Member

$\S$ Associate Professor, Department of Aerospace Engineering, University of Michigan, AIAA Associate Fellow
} 
wing from producing too much of a pitch-down moment, limiting the trim drag, it does not allow for a trade between wing aerodynamic performance and the trimming of the full aircraft. In addition, the aerodynamic load on the tail influences the circulation distribution of the whole aircraft in the Trefftz plane, directly influencing the induced drag. Moreover, the minimum induced drag for a given wing-tail system is not achieved with an elliptically loaded wing, since the optimal wing shape and twist are influenced by the horizontal tail design, so it is important to consider the whole system [5]. Thus, there is a need to consider the simultaneous analysis and design optimization of the the wing and tail when performing aerodynamic shape optimization.

To address this need, we perform a series of aerodynamic shape optimizations of the Common Research Model (CRM) configuration. We have previously established that Euler-based models yield non-physical designs for transonic wings [6], and thus we use RANS with a Spalart-Allmaras (SA) turbulence model exclusively. The investigations in this paper aim to answer several questions related to the trim of full configurations. The first is how different are the results obtained by optimizing the aircraft with trim constraints versus not, and also by considering horizontal-tail shape design versus not. The second question is how close can we get to the true optimum obtained by optimizing the full configuration by shaping the wing in the presence of the body only, and incorporating a trim penalty based on a value given by a trimmed drag polar of the whole configuration.

A large number of investigations have been published on design optimization of the wing only, together with a few others on optimization design of the wing within a more complex configuration like wing-body or wing-body-tail configurations. Among this previous research, however, the influence of the horizontal-tail shape and trimming have not been investigated in detail with RANS CFD. In this paper, we present the optimization results of the lift-constrained drag minimization of the CRM wing-body-tail configuration [7] using the RANS CFD, with shape optimization of both wing and tail simultaneously.

Aerodynamic shape optimization with gradient-based optimizers has been extensively investigated over the last few decades $[8,9,10,11,12,13,3,4]$. Various researchers have investigated a RANS-based single-point benchmark developed by the AIAA Aircraft Design Optimization Discussion Group (ADODG), which consists of shape optimization of the CRM wing alone $[3,14,15,16]$, but trim considerations for all these studies were limited to a pitch moment coefficient constraint.

There have been a few studies considering the trimmed CRM configuration in the context of aerostructural design optimization, where both the aerodynamic shape and structural sizing were optimized [17, 18]. Flying wing configurations, such as the blended wing-body (BWB), exhibit a strong coupling between the aerodynamic performance and trim, since the wing must be able to trim the moment on its own, while maintaining longitudinal stability [19]. The aerodynamic shape optimization of BWB configurations including trim and stability was studied by Lyu and Martins [4], as well as Reist and Zingg [20]. As for tail design optimization, several investigations addressed conceptual level design, such as the tail sizing, dihedral and load [5,21,22], but there have not been much studies on the aerodynamic shape optimization applied to the tail and wing simultaneously using RANS CFD technique on conventional configuration of commercial aero-transport to explore the merit for drag reduction.

In this paper, we choose the 4th Drag Prediction Workshop (DPW-4) CRM wing-body-tail configuration as our baseline model [7]. The reason for choosing this configuration is that the the DPW-4 CRM is representative of a conventional wide-body commercial transport aircraft, which includes a supercritical wing, a wing-body fairing, as well as an horizontal-tail. The horizontal-tail was designed to satisfy the typical stability and control requirements [7], so the DPW-4 CRM is suitable for this investigation.

This paper is organized as follows. The numerical tools used in this work are described in Section II. The problem formulation, baseline geometry, and CFD mesh are introduced in Section III. The single-point wing aerodynamic shape optimization without a trim constraint is presented in Section IV, while the optimization with the trim constraint is discussed in Section V. Then a single-point wing aerodynamic shape optimization without the tail, but with an estimated tail trim penalty is presented in Section VI. Finally, a simultaneous optimization of wing and tail shape optimization with and without the trim constraint is presented in Section VII and Section VIII.

\section{Methodology}

This section describes the numerical tools and methods we used for the optimization studies. These tools are components of the framework for multidisciplinary design optimization (MDO) of aircraft configurations with high fidelity $(\mathrm{MACH})[6,23,24]$. MACH can perform the simultaneous optimization of aerodynamic shape and structural 
sizing variables considering aeroelastic deflections [25]. However, in this paper we focus solely on the aerodynamic shape optimization without structural considerations.

\section{A. Geometric Parametrization}

Both the wing and the tail geometries are parametrized using the free-form deformation (FFD) volume approach [26]. The FFD volume parametrizes the changes of the embedded geometry rather than the geometry itself, resulting in a more efficient and compact set of geometry design variables, thus making it easier to manipulate complex geometries. Any geometry may be embedded inside the volume by performing a Newton search to map the parameter space to physical space. All geometric changes are performed on the outer boundary of the FFD volume. Any modification of this outer boundary can be used to indirectly modify the embedded objects. The key assumption of the FFD approach is that the geometry has constant topology throughout the optimization process, which is usually the case for aerodynamic design optimization. In addition, since FFD volumes are tri-variate B-spline volumes, the sensitivities of any point inside the volume can be efficiently computed. Figure 1 shows the FFD volume and the geometric control points for the aerodynamic shape optimization for the wing-body-tail configuration. The whole aircraft is enveloped by 28 FFD volumetric blocks, one of which parametrizes the wing and another parametrizes the tail. The wing is parametrized using 816 design variables that perturb the shape, which are the $z$-coordinates of the FFD control points. Another 144 design variables parametrize the tail. In the design cases that enforce the trim constraint, an additional design variable is used that rotates the tail as a solid body.The axis for the solid rotation of the tail is at the $40 \%$ chord length of the tail root section and is normal to the symmetry plane. As for the geometrical constraints, 1 wing volume constraint to constrain the wing internal volume to be no less than the initial one has been adopted, together with 1000 thickness constraints uniformed distributed on the wing (40 spanwise $\times 25$ chordwise) to constrain the wing thickness at each position to be no less than the initial one. Besides, the wing root incidence angle has been constrained to be fixed.

\section{B. Mesh Perturbation}

Since FFD volumes modify the geometry during the optimization, we must perturb the CFD mesh to solve for the modified geometry. The mesh perturbation scheme used in this work is a hybridization of the algebraic and linear elasticity methods [26]. The idea behind the hybrid warping scheme is to apply a linear-elasticity-based warping scheme to a coarse approximation of the mesh to account for large, low-frequency perturbations, and to use the algebraic warping approach to attenuate small, high-frequency perturbations.

\section{CFD Solver}

For the CFD, we use the SUmb flow solver [27], which is a finite-volume, cell-centered multiblock solver for the compressible Euler, laminar Navier-Stokes, and RANS equations (steady, unsteady, and time-periodic). SUmb provides options for a variety of turbulence models with one, two, or four equations and options for adaptive wall functions. The Jameson-Schmidt-Turkel (JST) scheme [28] is used for the spatial discretization. The main flow is solved using an explicit multi-stage Runge-Kutta method along with geometric multi-grid technique. A segregated Spalart-Allmaras (SA) turbulence equation is iterated with the diagonally dominant alternating direction implicit (DDADI) method. We have developed a discrete adjoint method for the Euler and RANS equations for the efficient computation of gradients required for optimizations [6]. The adjoint implementation supports both the full-turbulence and frozen-turbulence modes, but in the present work we use the full-turbulence adjoint exclusively. The adjoint equations are solved with preconditioned GMRES [29] using PETSc [30, 31, 32].

\section{Optimization Algorithm}

Because of the high computational cost of CFD solutions, it is critical to choose an efficient optimization algorithm that requires a low number of function calls. Gradient-free methods, such as genetic algorithms, have a higher probability of getting close to the global minimum for cases with multiple local minima, but slow convergence and the large number of function calls make gradient-free aerodynamic shape optimization infeasible with the current computational resources, especially for large numbers of design variables. Since we require hundreds of design variables, we use a gradient-based optimizer combined with adjoint gradient evaluations to solve the problem efficiently. The local minima issue of the gradient-based aerodynamic shape optimization has been explored by Lyu et al. [3], who concluded that 


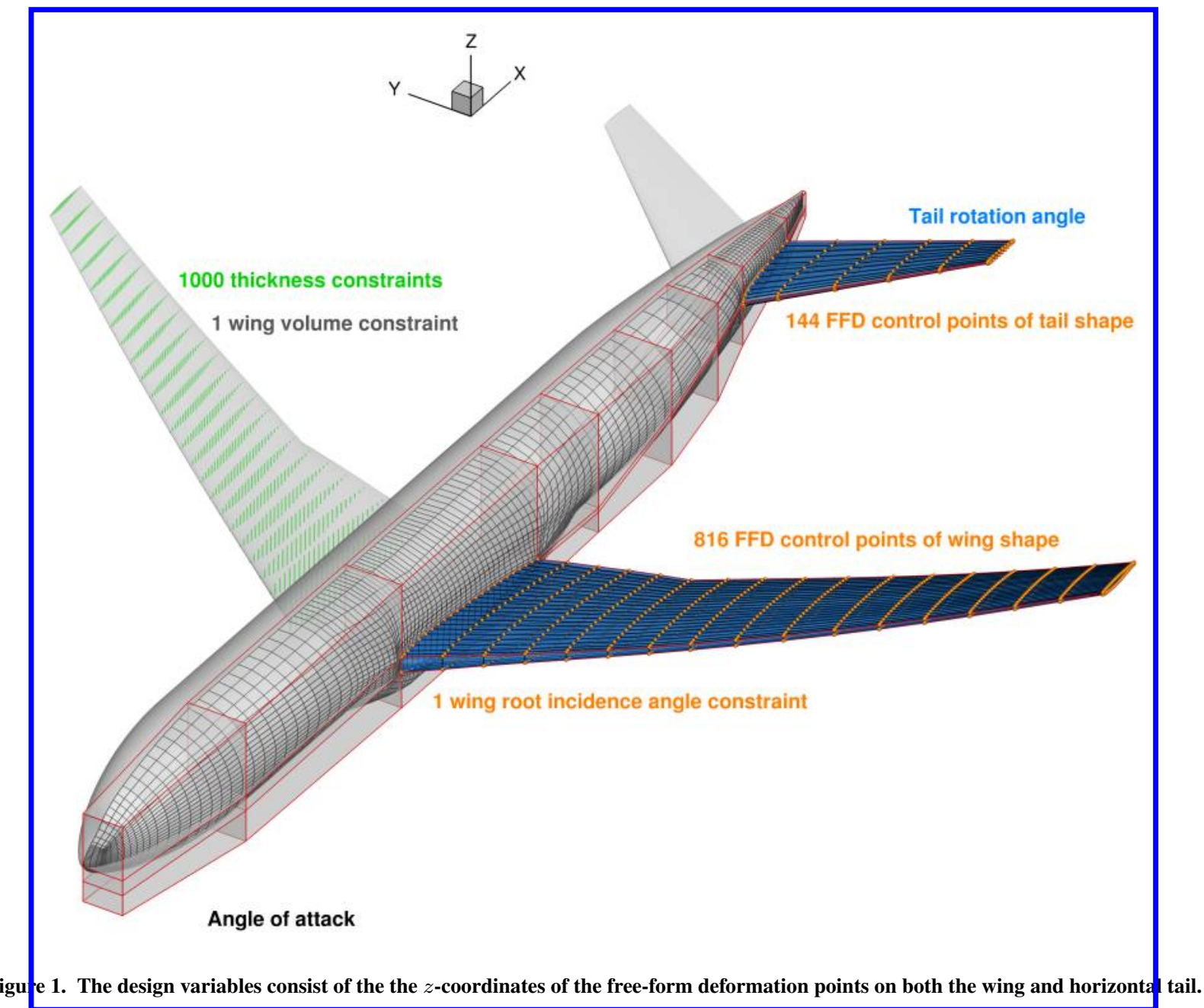

while there numerical local minima, these are restricted to a small space around the optimum, with differences in drag coefficient in the order of a fraction of a count.

The optimization algorithm we use is SNOPT (sparse nonlinear optimizer) [33] through the Python interface pyOpt [34]. SNOPT is a gradient-based optimizer that implements a sequential quadratic programming method; it is capable of solving large-scale nonlinear optimization problems with thousands of constraints and design variables.

\section{Problem Formulation}

We now present the details of the baseline geometry as well as the optimization problem formulations for the various cases that we solved.

\section{A. Baseline Geometry}

As previously mentioned, the baseline geometry is that of the DPW-4 CRM, which is a wing-body-tail configuration, with the tail rotation set to zero. The development of the CRM is detailed by Vassberg [7], and the geometry is shown in Fig. 2. The reference point is at $25 \%$ MAC, which corresponds to the position of the center of gravity.The coordinates for this point are $x=33.67786 \mathrm{~m}, y=0.007669 \mathrm{~m}$ and $z=4.51993 \mathrm{~m}$. The reference area is $191.8447776 \mathrm{~m}^{2}$ and the reference length(MAC) is 7.00532. The nominal flight condition of CRM is a cruise Mach number of $M=$ 
0.85 with a nominal lift coefficient of $C_{L}=0.50$. The Reynolds number is selected as 5 million based on the mean aerodynamic chord, which is consistent with the wing-tunnel test.

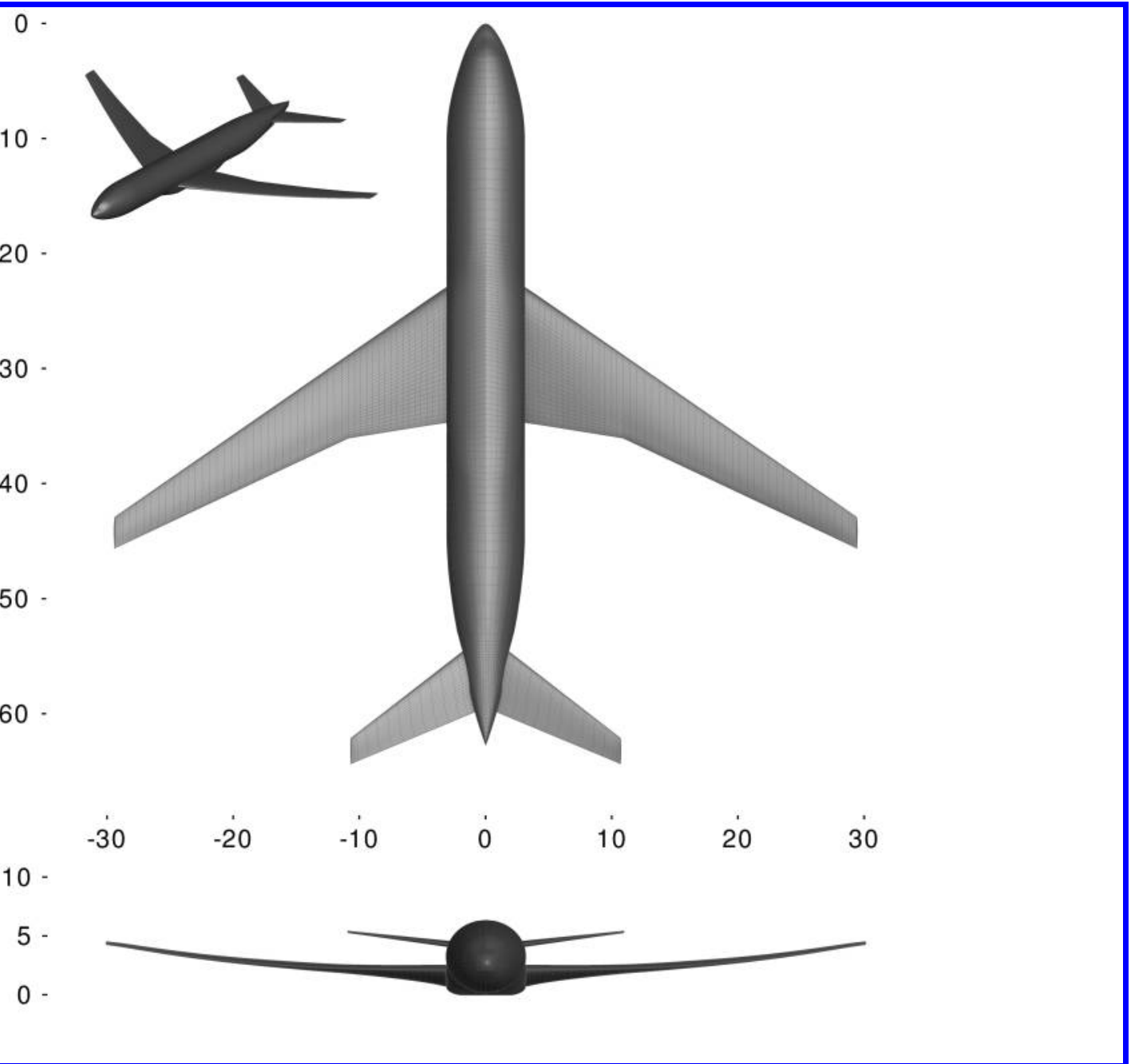

Figure 2. baseline geometry.

\section{B. Mesh Convergence Study}

The CFD structured mesh is generated with ANSYS ICEM-CFD, and consists of a multi-block structured nodematching mesh with O-H topology. The total number of the blocks in this mesh is 1018 . We first perform a mesh convergence study on this mesh with different coarsening levels. The flow condition for this mesh convergence study is the nominal cruise flow condition $\left(M=0.85, R e=5 \times 10^{6}\right.$. In optimization we use the same multi-grid-level methodology we used previously [35] to accelerate the design optimization. The finest mesh that is directly generated by ICEM-CFD has about 47.8 million nodes (denoted as the L0 mesh), and then this mesh is coarsened twice, resulting in a 5.97 million node mesh (L1) and a 746, 000 node mesh (L2). The surface and symmetry plane for the L0, L1, and $\mathrm{L} 2$ meshes are shown in Figure 3. The mesh size, $y_{\max }^{+}$values, $C_{L}$ values, $C_{D}$ values and $C_{M_{y}}$ values at the nominal operating condition for these three mesh levels are listed in Table 1. Besides, the Figure 8 shows the drag convergence

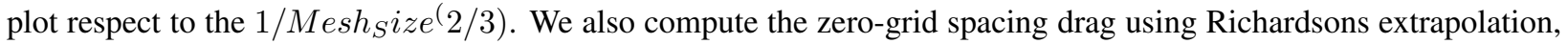
which estimates the drag value as the grid spacing approaches zero [36]. The zero-grid spacing drag coefficient is 267.8 counts for the baseline CRM wing. As shown in the Figure 8, the drag convergence curve is well linear and 
indicates that the mesh accuracy would be consistent while being refined. After considering the trade-off between computational time and accuracy, we decided to use the two coarser mesh levels (L1 and L2) for the optimizations. Since in the following cases we would perform optimization including tail rotating and tail shape changes, which could influence the longitudinal stability of the aircraft, so we computed $-\partial C_{M} / \partial C_{L}$ of the baseline geometry at the cruise condition, which is $30 \%$ and indicates that a static margin of $30 \% \mathrm{MAC}$ would be obtained when the center of gravity is at the location of the referenced point.

\begin{tabular}{rrrrrr}
\hline Mesh level & Mesh size & $C_{D}$ & $\boldsymbol{y}^{+}$ & $C_{L}$ & $C_{M_{y}}$ \\
\hline$h=0$ & $\infty$ & 0.026581 & & & \\
$\mathrm{~L} 0$ & $47,751,168$ & 0.027353 & 0.996 & 0.50000 & -0.0386 \\
$\mathrm{~L} 1$ & $5,968,896$ & 0.029068 & 2.744 & 0.50000 & -0.0411 \\
$\mathrm{~L} 2$ & 746,112 & 0.035227 & 5.244 & 0.50000 & -0.0508 \\
\hline
\end{tabular}

Table 1. Mesh convergence study

\section{Optimization Problem Formulation}

The objective of the optimization problem is set to minimize the drag coefficient of the aircraft, subject to a lift constraint $\left(C_{L}=0.5\right)$. As previously mentioned, the shape design variables are the $z$-coordinates of 816 control points of the FFD volume enveloping the wing, and 144 control points of the FFD volume enveloping the tail. The other two design variables are the horizontal tail rotation angle and the angle of attack. The FFD control points are distributed on the FFD volume surface in a regular grid with 17 spanwise and 24 chordwise points, with two layers controlling the upper and lower surfaces separately, as shown in Fig. 1. Since the transonic wing aerodynamic performance is sensitive to the leading edge shape, the chordwise FFD control points are not distributed uniformly: there is a denser at the leading edge to set allow more freedom in the leading edge shape. We also add geometric constraint to control the geometry in order to get a practical design. The geometric constraints are 1000 thickness constraints imposed at 25 chordwise and 40 spanwise locations of the wing, as shown in Fig. 1. These thicknesses are enforce to be no less than the thickness of the baseline at each location. Another geometric constraint is the wing internal volume constraint, which is set to be no less the initial value.

\section{Surface Sensitivity on the Baseline Geometry}

We first performed a sensitivity analysis of the initial geometry at the nominal cruise condition to examine the sensitivities of the initial geometry. Figure 4 shows the derivatives of $C_{D}$ with respect to shape variations in the $z$ direction on the wing and fuselage. The sensitivity of the drag with respect to the wing surface shape can be visualized through the sensitivity contour plot; the value at each surface mesh node is the amount of drag reduction with unit displacement of the mesh node in $z$ direction. In Fig. 4, we can see that the regions with relatively high sensitivity of $C_{D}$ are the shock wave region on the wing upper surface. This is expected, since shaping in this are could drastically reduce the wave drag, and indicates that the optimization will try to eliminate the shock at the beginning in order to reduce $C_{D}$. Another sensitivity contour is shown in Figure 5, which plots the derivatives of $C_{M_{y}}$ with respect to shape variations on the tail in the $z$ direction. The derivatives distribution on the tail indicated that the tail shape changes towards positive lift would lead to a larger negative pitching moment. These sensitivity plots are only a linearization about the initial design point, and they provide no information about the constraints. Nonetheless, these sensitivity contours indicate what drives the design at this initial point.

\section{E. Summary of the Cases}

Table 2 summarizes the cases have been researched in this paper, the objectives of all the cases are minimization of the drag coefficient $C_{D}$. First, we optimized the wing-body-tail configuration with wing shape design variables, then in the second case, we added tail rotation as another design variable with trimming constraint. In the third case and the fourth case, we used the wing-body configuration by taking off the tail from the original wing-body-tail, one is with $C_{L}$ and $C_{M}$ constraints using an surrogate model estimation, the other is with $C_{L}$ and $C_{M}$ fixed at the values of 


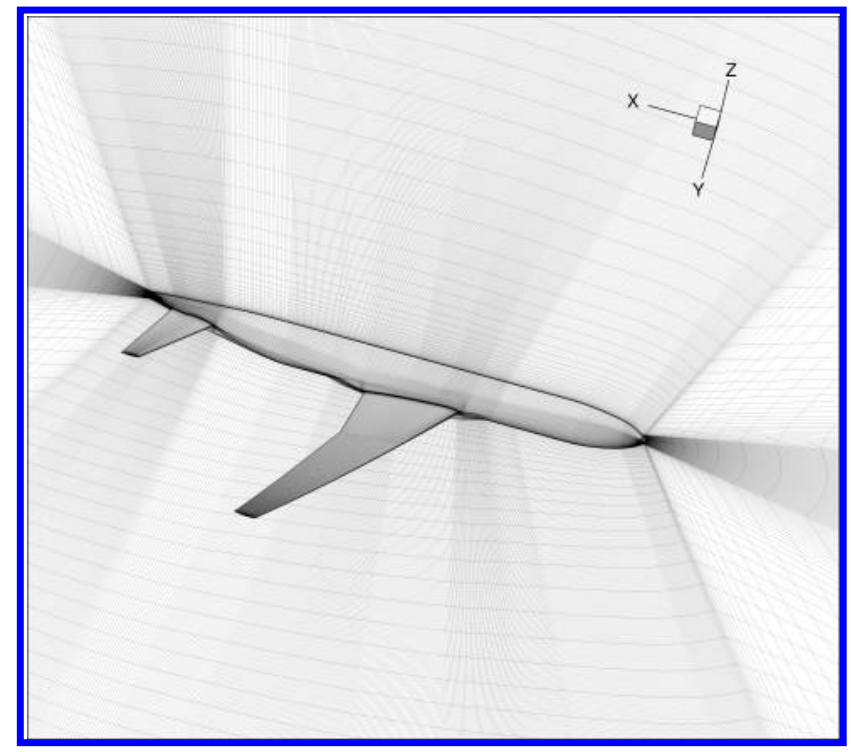

(a) L0 mesh: $47.8 \mathrm{M}$ cells, 273.53 drag counts

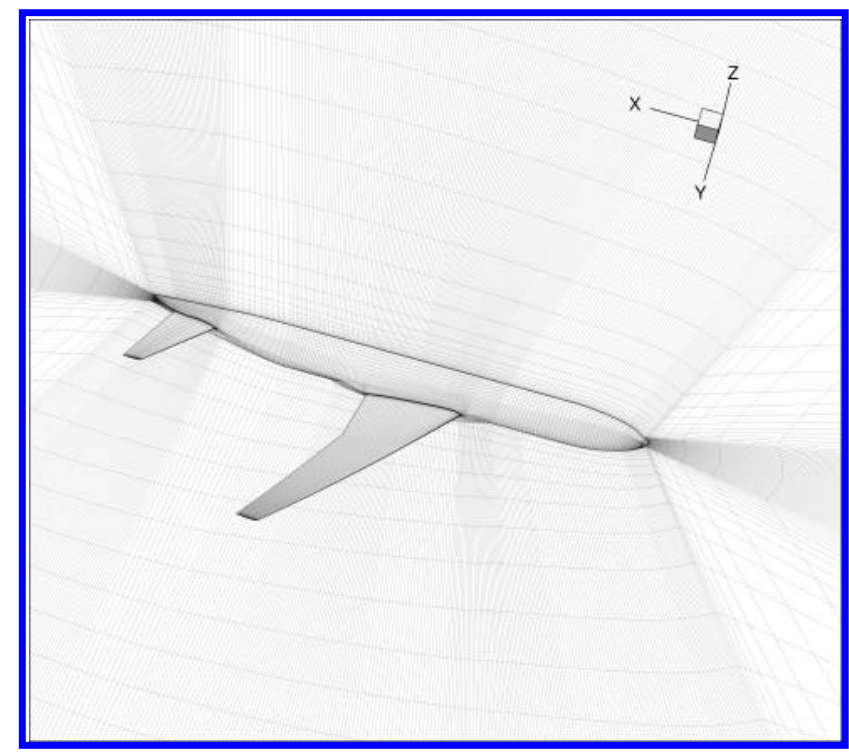

(b) L1 mesh: $5.97 \mathrm{M}$ cells, 290.68 drag counts.

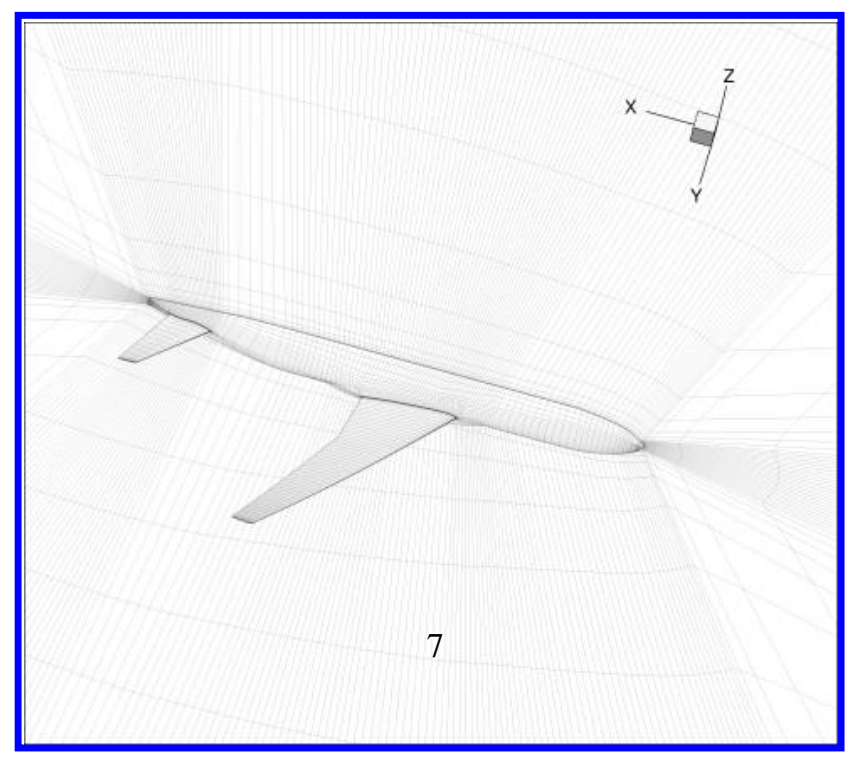

(c) L2 mesh: 746, 112 cells, 352.27 drag counts.

Figure 3. O-H meshes of varying sizes were generated using ICEM-CFD. 


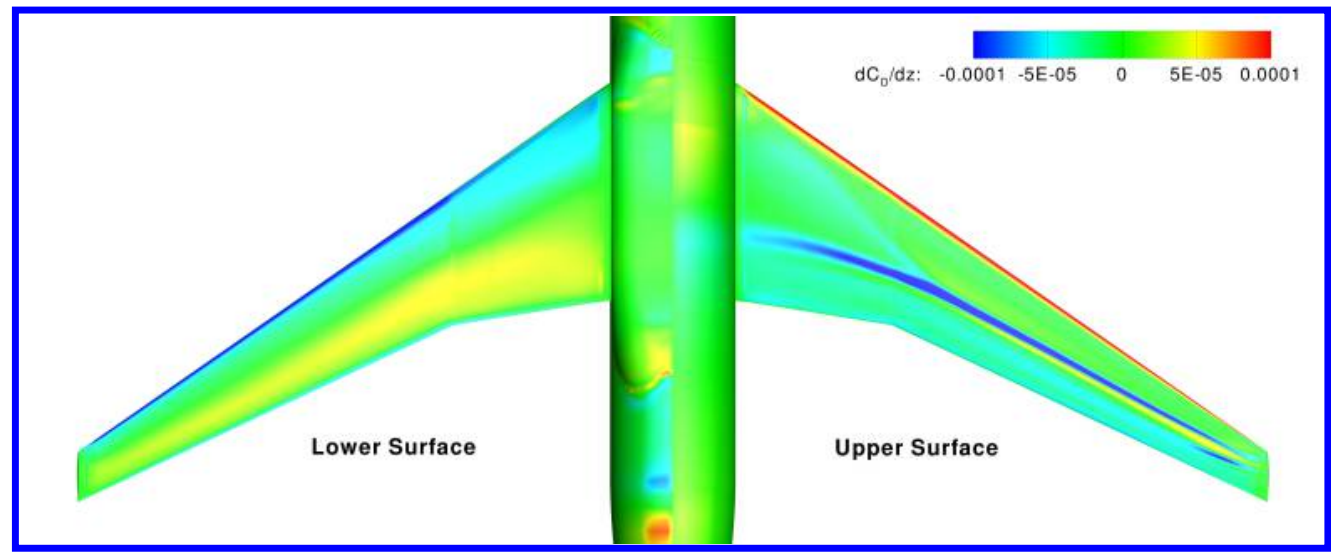

Figure 4. Drag coefficient sensitivity contour of the initial configuration with respect to wing and fuselage shape variables.

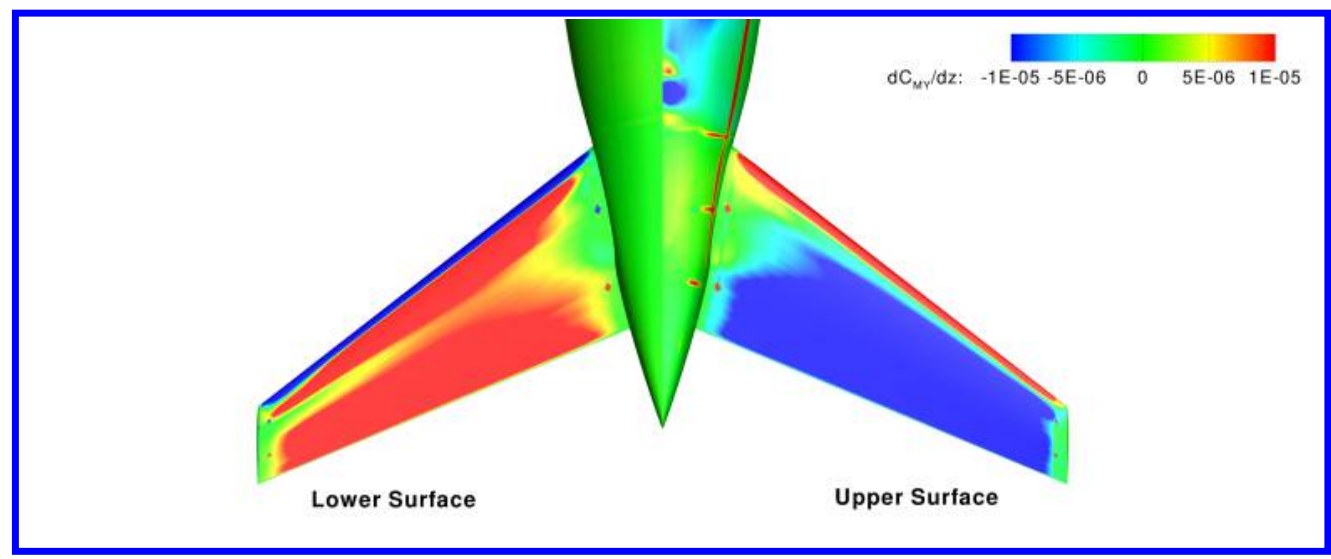

Figure 5. Aircraft pitching moment sensitivity contour of the initial aircraft with respect to horizontal tail shape variables.

wing-body component from the trimmed baseline geometry. Finally, we further added the tail shape design variables to the wing-body-tail configuration optimization with and without trimming constraint. 


\begin{tabular}{|c|c|c|}
\hline Configuration & Design variables & Constraints \\
\hline wing-body-tail & AoA, wing shape & $\begin{array}{l}\text { Geometric } \\
C_{L}=0.5\end{array}$ \\
\hline wing-body-tail & $\begin{array}{l}\text { AoA, wing shape } \\
\text { tail rotation }\end{array}$ & $\begin{array}{l}\text { Geometric } \\
C_{L}=0.5, C_{M}=0\end{array}$ \\
\hline wing-body & AoA, wing shape & $\begin{array}{l}\text { Geometric } \\
C_{L}=0.5, C_{M}=0 \text { (Estimated) }\end{array}$ \\
\hline wing-body & AoA, wing shape & $\begin{array}{l}\text { Geometric } \\
C_{L}=0.5256, C_{M} \geq-0.0996\end{array}$ \\
\hline wing-body-tail & $\begin{array}{l}\text { AoA, wing shape } \\
\text { tail shape }\end{array}$ & $\begin{array}{l}\text { Geometric } \\
C_{L}=0.5\end{array}$ \\
\hline wing-body-tail & $\begin{array}{l}\text { AoA, wing shape } \\
\text { tail shape, tail rotation }\end{array}$ & $\begin{array}{l}\text { Geometric } \\
C_{L}=0.5, C_{M}=0\end{array}$ \\
\hline
\end{tabular}

Table 2. Summary of the all drag minimization cases.

\section{Untrimmed Aerodynamic Shape Optimization of the Wing}

In this section, we present the results of the single-point aerodynamic shape design optimization of the CRM wing-body-tail configuration, where only wing shape design variables are varied with no enforcement of a moment or trim constraint. Two mesh levels (L2 with 746, 112 cells and L1 with 5.97 million cells) were used in the multilevel optimization. This case is run with 64 processors on the L2 mesh and 256 processors on the L1 mesh. The design variables in this case are the wing shape design variables and the angle of attack, with the constraints of lift coefficient and geometric constraints. The tail rotation is not a design variable in this case, since we make no attempt to trim the configuration. The optimized wing has $3.54 \%$ lower drag on L1 mesh compared to that of the initial geometry. The drag decreased from 290.7 counts to 280.4 counts at the nominal flow condition. The negative pitching moment coefficient increased from -0.041 to -0.078 , and the angle of attack is increased from 2.4 degrees to 3.1 degrees. Figure 7 shows the comparison between the initial and the optimized wings. The convergence history of the optimization is shown in Figure 6 for the two-level optimization process. 

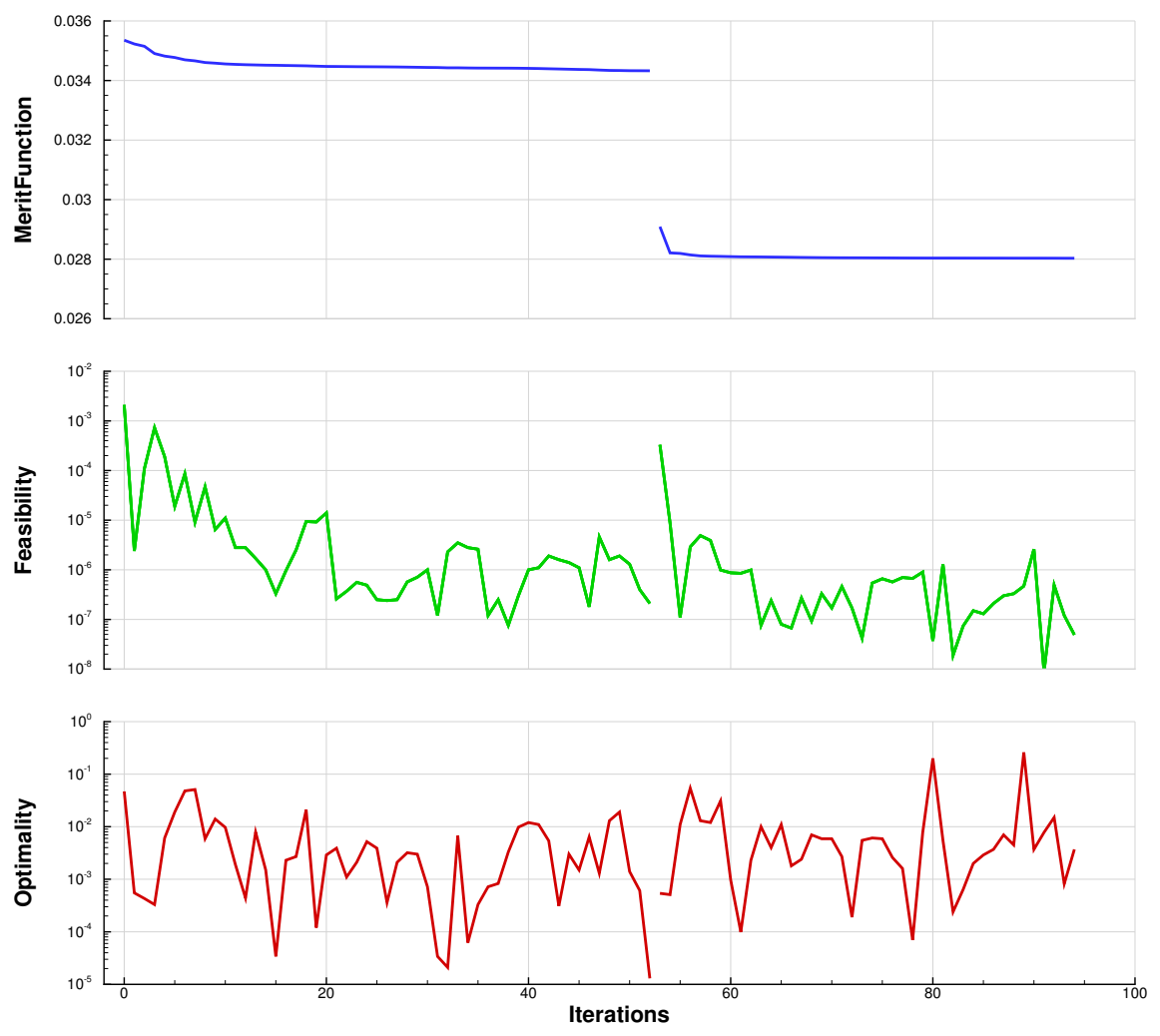

Figure 6. Convergence history for the single-point untrimmed case.

On the left side of Fig. 7 is the comparison of upper surface pressure coefficient contour, in which the the baseline wing exhibits a front of closely spaced pressure contour lines spanning a significant portion of the wing, indicating a shock wave. On the other side, the optimized wing exhibits parallel pressure contour lines with roughly equal spacing, indicating a nearly shock-free solution. Below the contour comparison plot, the front view shows the shock region, where a large region of shock is rendered as orange above the upper surface of the baseline wing. The optimized wing does not show a shock. On the bottom-left side of Fig. 7, we show a comparison of the relative thickness, wing twist distribution, and normalized lift along the span.

On the right side of Fig. 7, we show the comparison of multiple airfoil section geometries and their corresponding pressure coefficient distribution, in which the red stands for the baseline wing and the blue for the optimized wing. These plots confirms that the shock has been eliminated after optimization, with the upper surface pressure recovering smoothly from leading edge to trailing edge in optimized wing, in contrast with the shock present on the baseline wing. Since no moment constraint is imposed in this case, and we did not have the tail rotation as design variable, the negative pitching moment is increased from -0.041 to -0.078 . The lift distributions of the optimized wing and the baseline wing are shown by separating the lift on wing-body and the tail. It shows that both the negative lift on the tail and the positive lift on the wing have decreased. In addition, the angle of attack increased from 2.41 degrees to 3.1 degrees.

From the results obtained in this first optimization, we conclude that optimization on the wing only without tail trimming could obtain a shock free design with a considerable amount of drag reduction. However, the negative pithing moment increased considerably, which means that it would lead to a higher drag if we trimmed this configuration. 


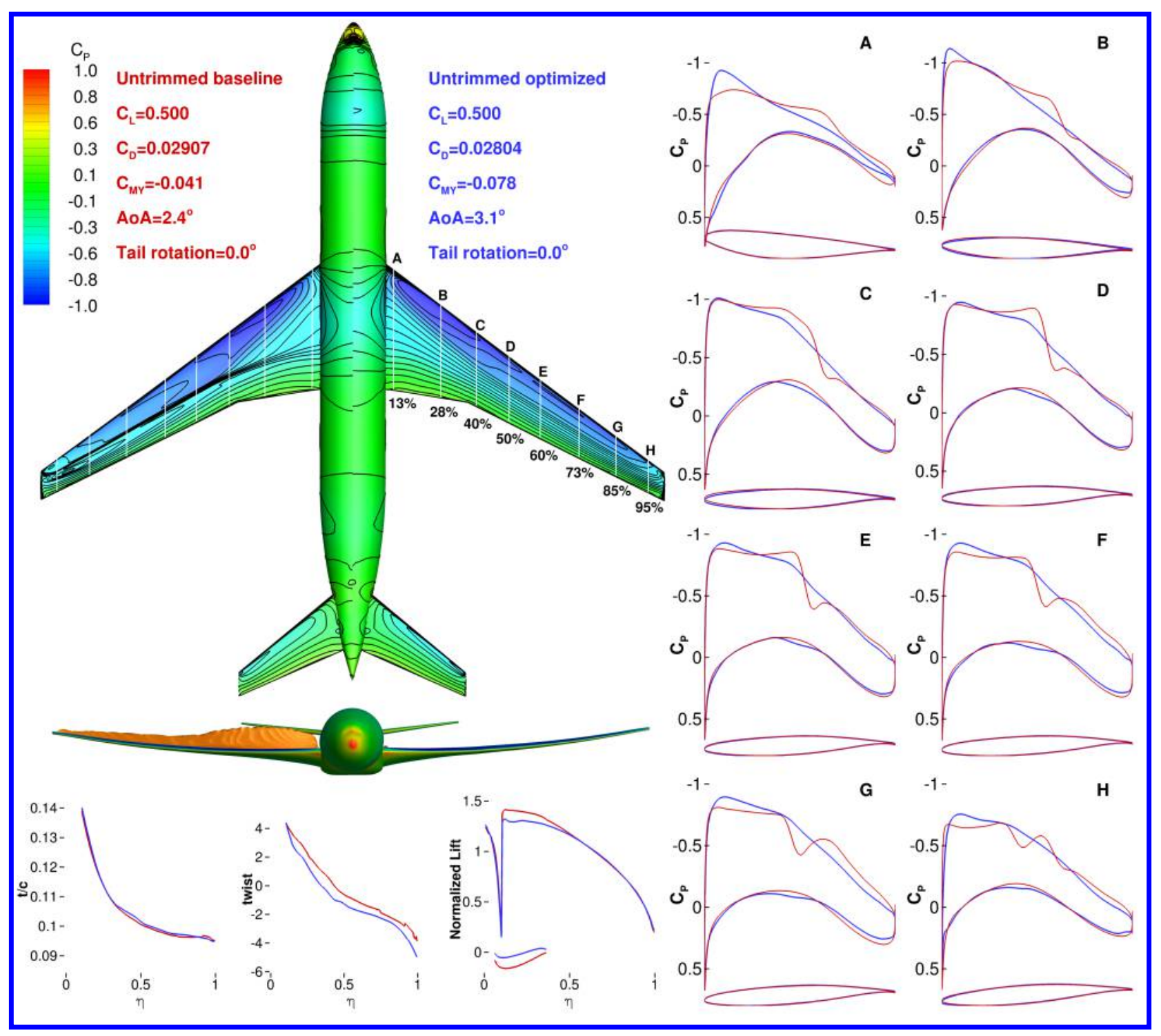

Figure 7. Comparison of the single-point untrimmed optimization with the baseline geometry.

\section{Trimmed Aerodynamic Shape Optimization of the Wing}

We perform another optimization case with a trim constraint by adding the tail rotation angle as an additional design variable. Figure 9 shows the comparison of the trim-constrained optimized wing with the previous untrimmed optimized wing. The optimized tail rotation angle for the trimmed optimization is -1.00 degree. The drag value of the trimmed optimization increased by 2.2 drag counts relative to the untrimmed optimized wing, which is still lower than that of the baseline. The angle of attack increased from 3.1 degrees to 3.4 degrees. The pressure coefficient contour of this trim-constrained optimal wing is similar to that of the untrimmed one, which also shows a shock-free optimized solution. From the section pressure coefficient plots we can see that the suction peak of inboard sections increased, which is consistent to increasing lift on the inboard wing to partially compensate for the increased negative lift on the tail required to trim the configuration. Besides, we did the drag convergence study on this optimized geometry, for comparison with the baseline geometry. Figure 8 shows this comparison, noted that this optimized geometry is eventually optimized at the L1 mesh, so the amount of drag reduction at L2 is even smaller than that at L1, mostly due 
to the low resolution of the relatively coarse mesh. However, the Figure 8 also shows that, the amount drag reduction of the optimized geometry has been maintained from L1 mesh to L0 mesh, which presents a drag value of 265.6 counts for L0 and 259.9 counts for aero-spacing mesh, and proves that the merit by optimization on the L1 mesh could still be obtained at a much refiner mesh.
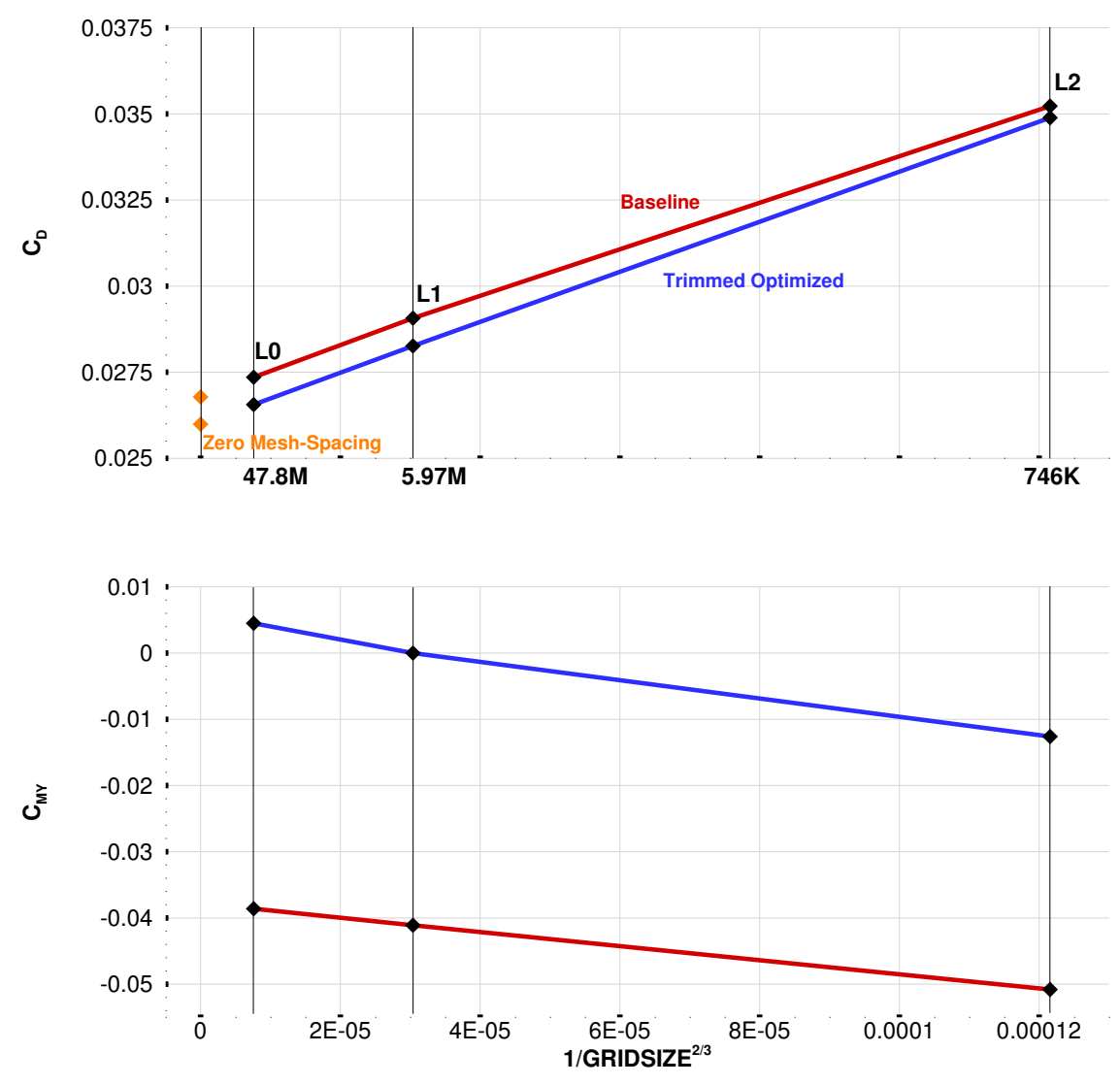

Figure 8. The mesh convergence for drag and moment coefficients

In Fig. 10, we compare the trimmed optimized wing with the baseline configuration, where this baseline configuration is now trimmed by rotating the tail. We can see that the drag reduction of the optimized configuration is even better relative to this trimmed baseline (12.1 drag counts lower, or $4.1 \%$ ). We also computed the $-\partial C_{M} / \partial C_{L}$ for this trimmed optimized configuration, which is $36.52 \%$, slightly higher than the baseline value, which is $30 \%$.

\section{Aerodynamic Shape Optimization of the Wing with Trim Penalty}

The goal for this case is to investigate how accurate it is to perform aerodynamic shape optimization on a wingbody configuration without the horizontal-tail, while adding a trim drag penalty proportional to the pitching moment of the tailless configuration.

First, we constructed the trim penalty surrogate model by performing CFD computations on the wing-body-tail configuration with series of tail rotation angles for a fixed lift coefficient $\left(C_{L}=0.5\right)$. By analyzing the forces acting on the separate components (wing, body and tail) we established how much drag and lift is required on the tail to trim the wing-body. By solving the flow with tail rotation ranging from -10 to 10 degrees, the relation of the lift and drag with respect to the moment was constructed using 1-D B-spline interpolation, as shown in Fig. 11. In the following 


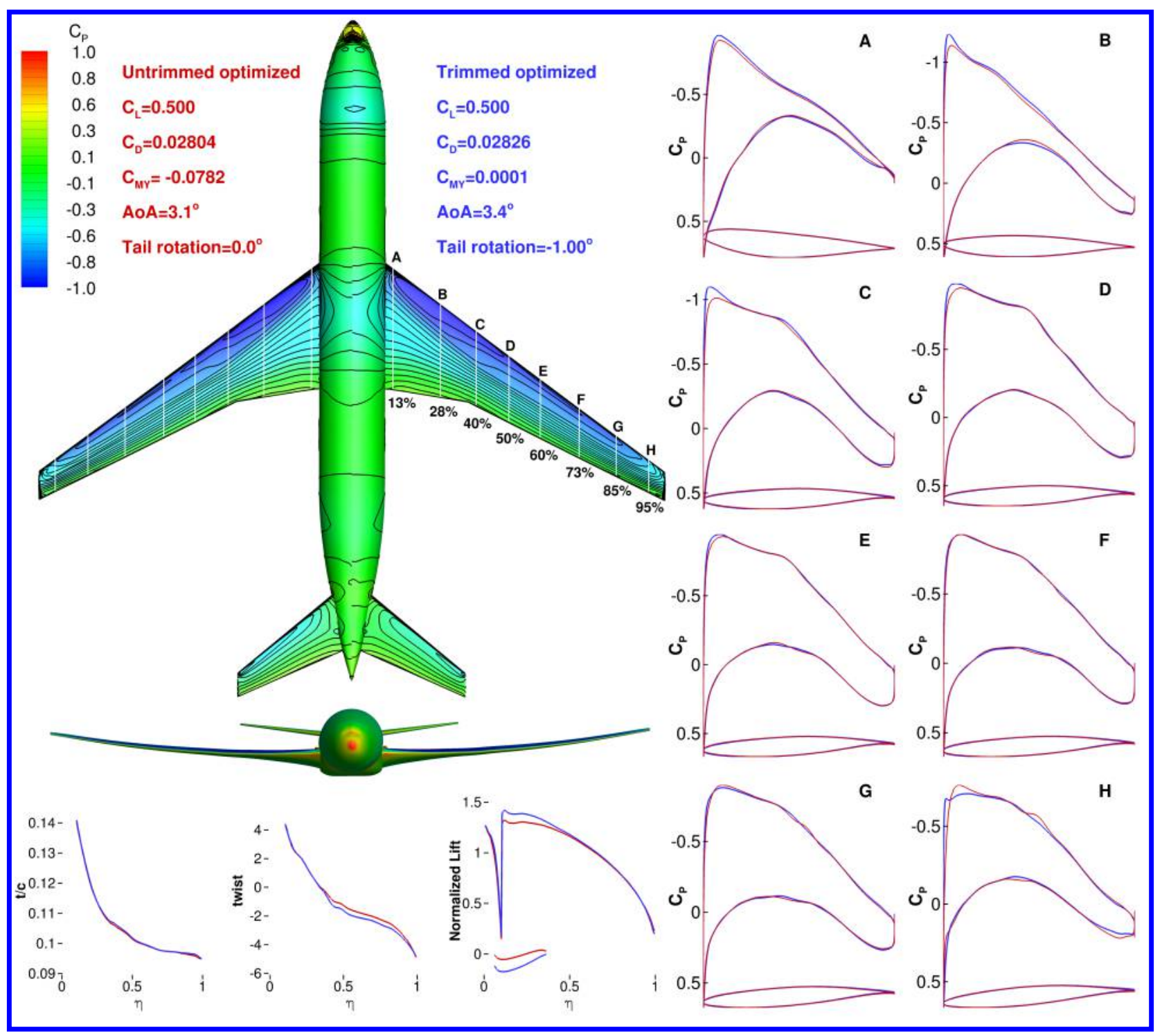

Figure 9. Comparison of the single point trimmed optimal configuration with the untrimmed configuration.

content, we denote the moment generated on the whole wing-body-tail configuration and $C_{M_{y}}^{\text {wbt }}$, the moment on the wing-body as $C_{M_{y}}^{w b}$, and on the moment of the tail as $C_{M_{y}}^{t}$. We use the same superscript convention for the drag and lift coefficients. So with the 1-D B-spline interpolation described above, we constructed the trim penalty surrogate model, which uses the moment generated on the tail to estimate the drag and lift on the tail. We denote this model as,

$$
\left\{\begin{array}{l}
C_{D}^{t}=f_{C_{D}}\left(C_{M_{y}}^{t}\right) \\
C_{L}^{t}=f_{C_{L}}\left(C_{M_{y}}^{t}\right)
\end{array}\right.
$$

In Fig. 11, we can see that the pitching moment generated by the tail varies linearly with the tail lift, and that the drag on the tail is nonlinear with respect to the moment. In particular, within the interval $[0,0.2]$, the tail drag decreases as the moment on the tail increases, and the minimum drag on the tail with respect to moment on the tail is approximately at $C_{M_{y}}=0.2$. In addition, since the tail is in the down-wash of the wing, for investigating whether this 


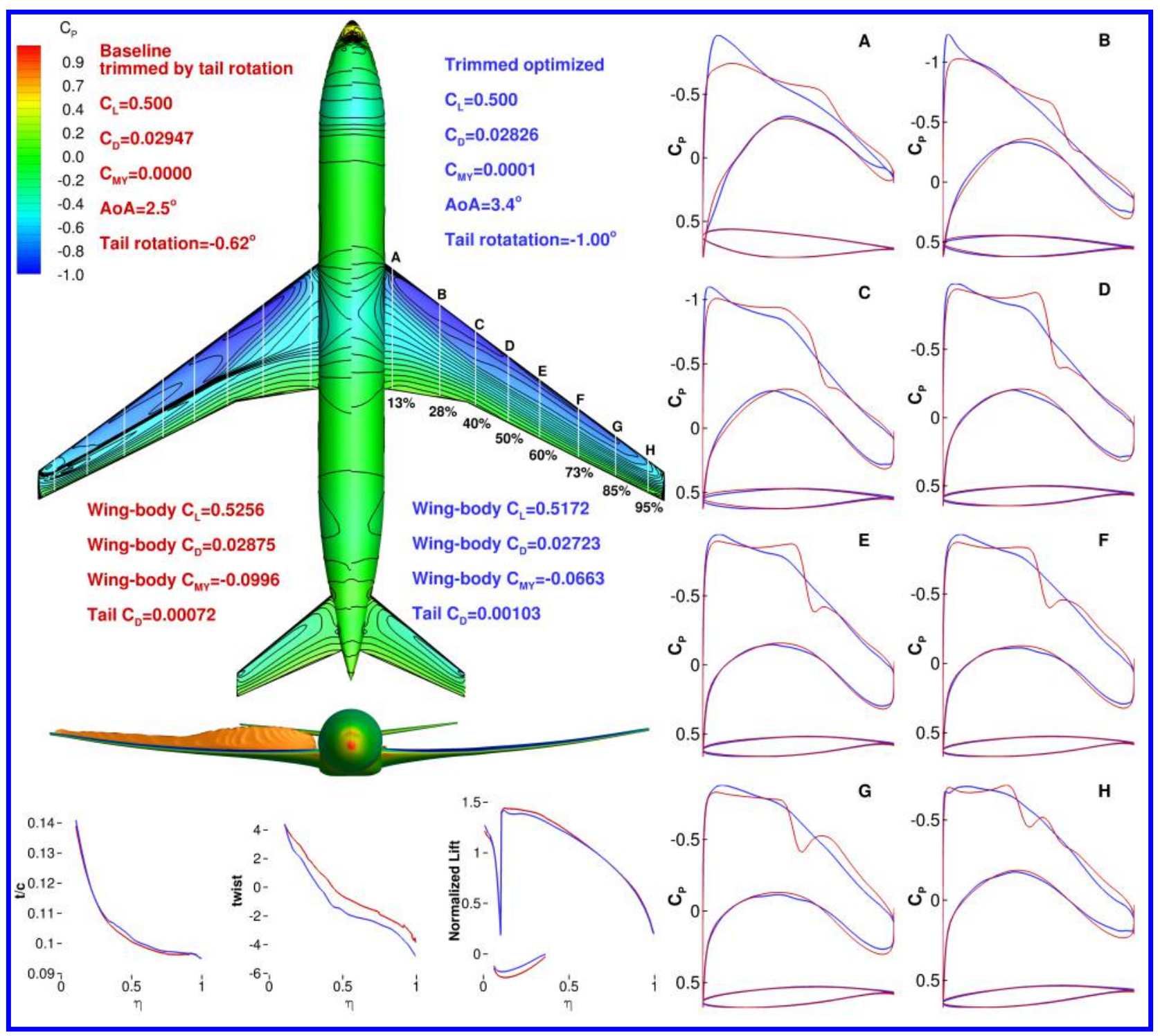

Figure 10. Comparison of the single point trimmed optimal configuration with the trimmed baseline.

trim penalty relationship would be sensitive to the wing as the wing lift distribution is changed after being optimized, we also compute this trim penalty data of the trimmed optimized configuration of the previous section. And the comparison between these two sets of data is showed, which turns out that the trim drag penalty of the optimized one matches that of the baseline well within the interval $C_{M_{y}}=[0,0.2]$, which is the normal range for the wing-body at the cruise condition. The difference in drag is approximately 1 drag count.

Besides, we quantify the tail rotation angles versus the tail moment on the tail. The rotation angle for the optimized configuration shifts downward relative to the baseline, and the difference is approximately 1 degree. In the previous section we established that the cruise angle of attack of the optimized configuration is 3.4 degrees, which is 1 degree higher than the baseline. This 1 degree difference in tail rotation angle is reasonable, since the actual angle of attack of the tail should be the sum of the the aircraft angle of attack and its rotation angle with influence of wing down-wash.

Given this surrogate model of the trim penalty versus required moment to trim the wing-body, we can optimize the wing-body without the tail. However, this does include the trimming influence of the horizontal-tail by computing the 
Baseline Data
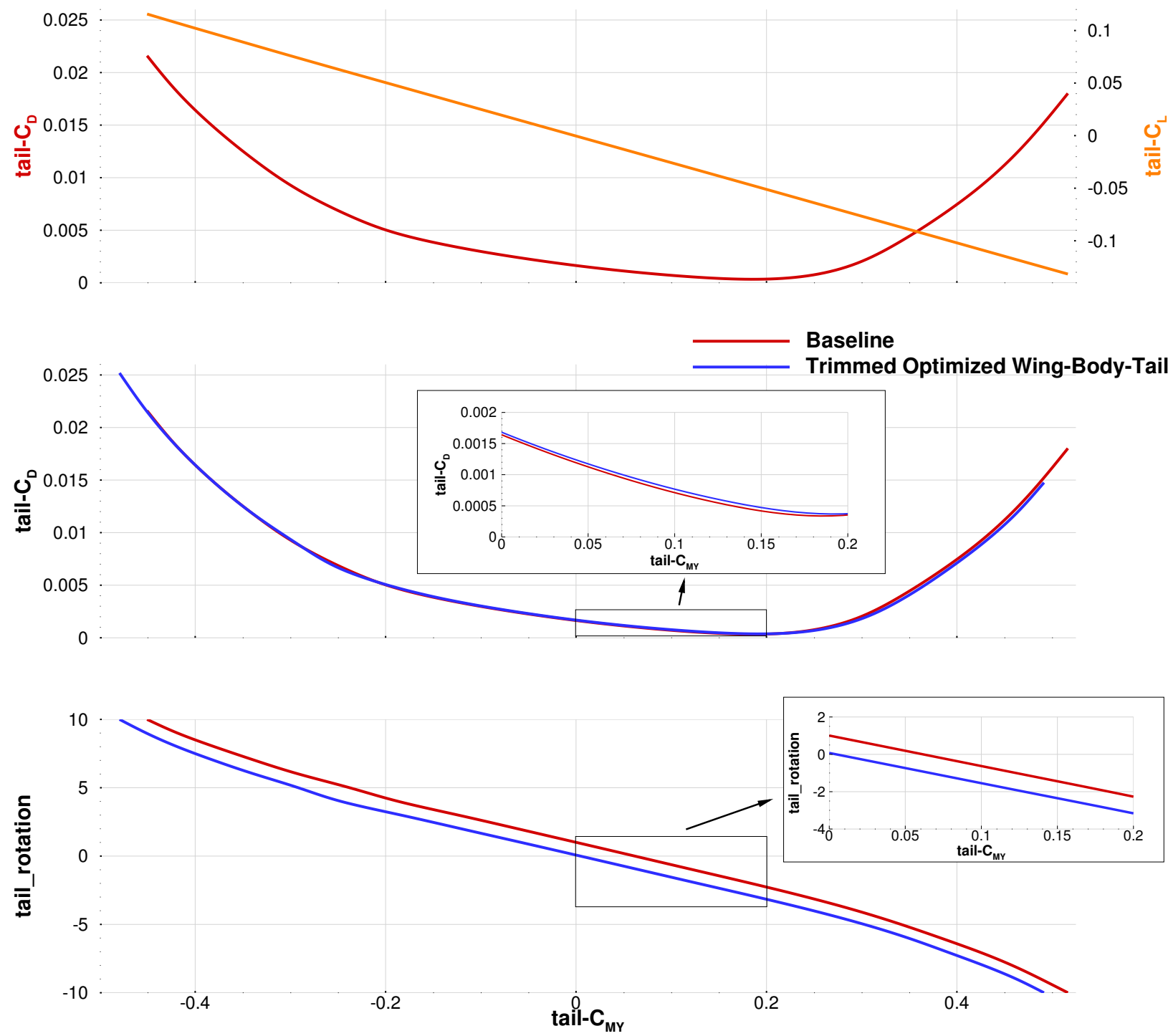

Figure 11. Tail drag and lift coefficients versus tail moment for the baseline configuration for a fixed total lift coefficient $\left(C_{L}=0.5\right)$ and comparison with the trimmed optimized one.

lift and drag on the tail with the model described above. For the whole wing-body-tail configuration, the objective of drag minimization and the lift and trim constraint would be denoted as Eqn. (2).

$$
\begin{array}{cl}
\min & C_{D}^{w b t} \\
\text { s.t. } & C_{L}^{w b t}=0.5 \\
& C_{M_{y}}^{w b t}=0.0
\end{array}
$$

When optimizing the wing-body, with the surrogate model that estimates the drag and lift on the tail, we transform the formulas above to be as in Eqn. (3). For instance, if the target lift coefficient of the full aircraft with the tail is 0.5 , then the constraint for the wing-body would be that the sum of the wing-body lift and the tail lift would be 0.5 in total. The tail lift would be computed using the model above at the moment value that trims the wing-body, which should 
be the negative of the moment on the wing-body. When optimizing the wing-body, the same mesh for wing-body-tail is used with hollowed tail blocks to maintain the same mesh topology as much as possible for a fair comparison. Figure 12 shows the mesh near the tail of the fuselage with and without the tail.

$$
\begin{aligned}
\min \quad C_{D}^{w b}+C_{D}^{t} & \\
\text { s.t. } \quad C_{L}^{w b}+C_{L}^{t} & =0.5 \\
C_{M_{y}}^{w b}+C_{M_{y}}^{t} & =0.0 \\
C_{D}^{t} & =f_{C_{D}}\left(C_{M_{y}}^{t}\right) \\
C_{L}^{t} & =f_{C_{L}}\left(C_{M_{y}}^{t}\right)
\end{aligned}
$$

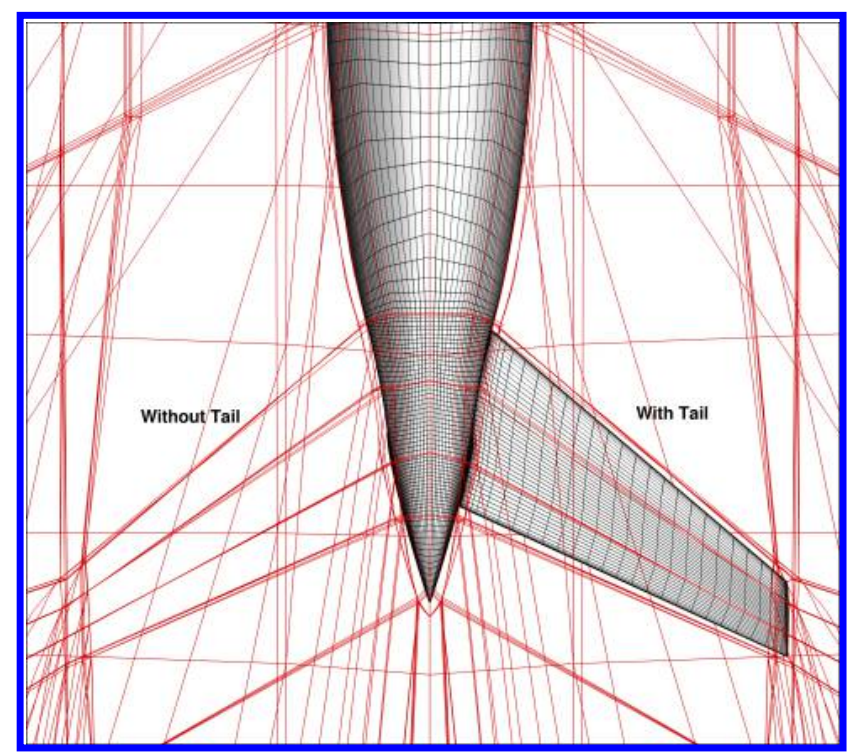

Figure 12. Comparison of mesh around the tail for the wing-body and wing-body-tail.

Following the strategy described previously, we perform optimization on the wing-body configuration without the tail, but with the surrogate model estimating the tail drag penalty. Then the optimized wing-body with this trim penalty is compared to itself by adding the tail back on and trimming it. Since we cannot use the estimated tail rotation angle after the wing is optimized, we solve the flow of this optimized wing-body with the tail back on at the $\operatorname{target} C_{L}=0.5$ with rotated tail to obtain a new set of data corresponding to this optimized wing-body geometry. For comparing the total drag, we recompute the trim penalty data for the optimized wing-body with the tail added back on. From the Figs. 13, we can see that the drag penalty is different from the previous two. Within the range $C_{M_{y}}=[0.05,0.20]$, the drag on the tail of the optimized wing-body is actually higher than both the baseline and trim optimized wing-body-tail configurations, while within the range $C_{M_{y}}=[0.00,0.05]$, it is approximately in the middle of the other two curves. Figure 14 shows the comparison of the optimized wing-body with estimated drag penalty with itself with the tail back on. Though the pressure distributions on the two wings are almost the same, the pressure contours on the aft-bodies are significantly different. Thus, we believe that the difference in the forces comes primarily from the aft-body.

Then we further compared the optimized wing-body with trim penalty by adding the tail back on, with the trimmed optimized wing-body-tail in the previous section. Figure 15 shows this comparison, where we can tell that the pressure distribution at each section is very similar. The one optimized with the trim penalty still has about 1.2 more counts in total drag compared to the trim-constraint optimized one.

We also compared the optimized wing-body-tail without the trim constraint and trimmed by rotating the tail after optimization, with the trim-constraint optimized wing-body with tail back on.Figure 16 shows this comparison. Although the optimized wing-body-tail without the trim constraint achieved a low drag value of about 280.4 counts, after 

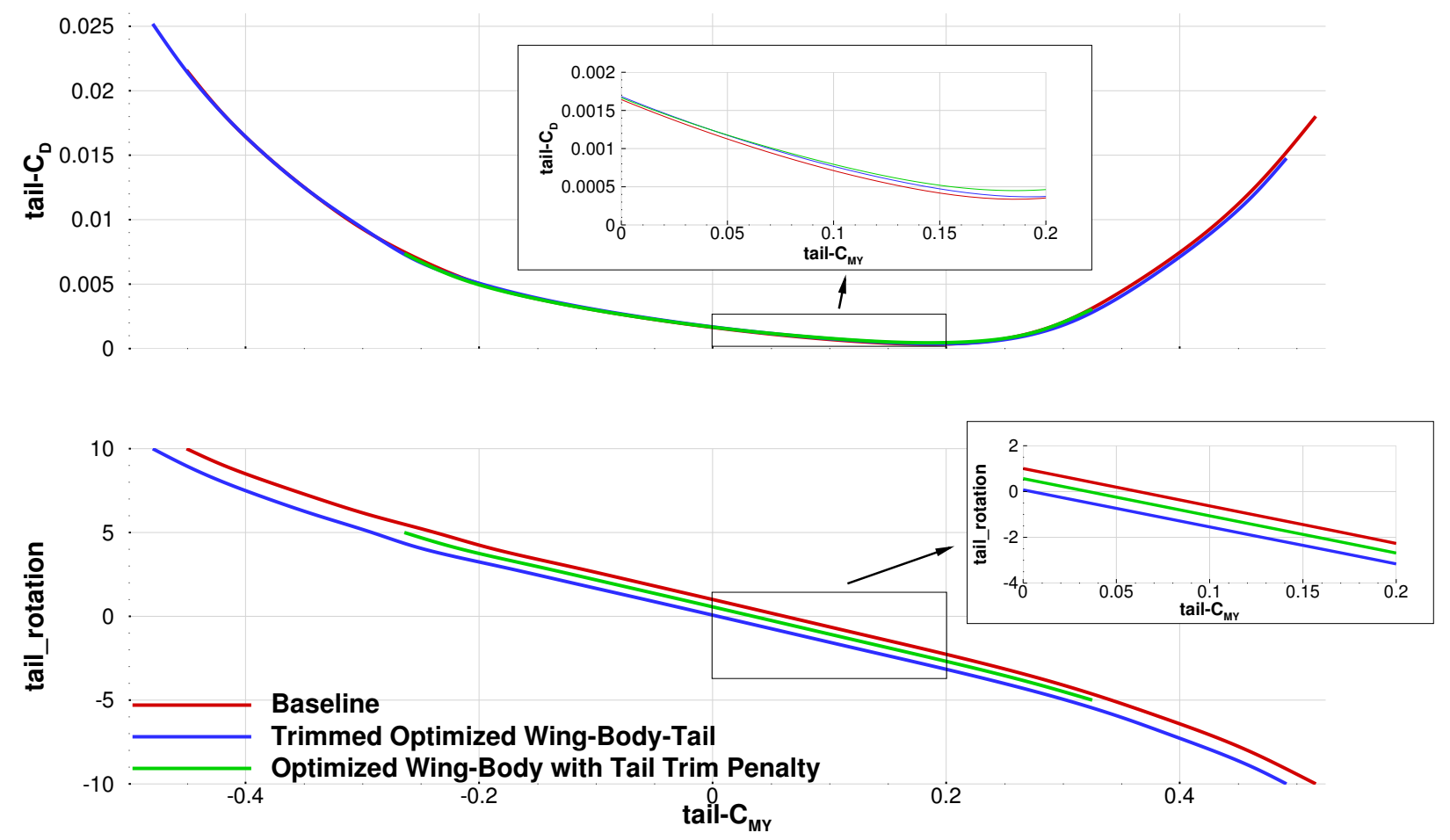

Figure 13. Tail drag and lift coefficients versus tail moment for the baseline configuration for a fixed total lift coefficient $\left(C_{L}=0.5\right)$ and comparison with the trimmed optimized one and optimized wing-body with tail trim penalty one.

trimming it by the tail rotation, the drag increased to 285.6 drag counts. Most of this increase in drag comes from the wing-body and not the tail, as seen in Fig. 16.

We also developed a design optimization case to investigate whether we can achieve a lower drag for the trimmed wing-body-tail by optimizing the wing-body without the tail with carefully selected lift and moment constraint values. To this end, we perform another optimization on the wing-body without the tail with the $C_{L}$ fixed at the value for the wing-body component of the trimmed wing-body-tail baseline, which is $C_{L}=0.5256$, and with $C_{M_{y}} \geqslant-0.0996$, which is the moment coefficient value for the wing-body component of the trimmed wing-body-tail baseline. Figure 17 shows the comparison of this optimization with the same trimmed optimized configuration with the tail. The comparison shows that the trimmed optimized wing-body-tail still has the lowest drag value, which is about 1.4 drag counts lower than that of the optimized wing-body with the new lift and moment constraints after adding the tail on. In addition, the comparison of normalized lift shows that the lift on the optimized wing-body one is higher than that of the trimmed optimized wing-body-tail one, which makes it harder for the optimizer to fully eliminate the shock.

From the results presented in this section, we conclude that for, single-point aerodynamic shape optimization, the lowest drag is achieved by the the trim-constrained wing-body-tail optimization, where the tail rotation is optimized simultaneously with the wing shape. Optimizing the wing-body without the tail, however with an estimated tail trim penalty or carefully chosen fixed lift and moment coefficient constraints can also achieve a low drag, but this is still 1-3 drag counts higher than the best.

\section{Simultaneous Aerodynamic Shape Optimization of the Wing and Tail}

To quantify the gains that could be obtained by optimizing both the wing and horizontal-tail shapes simultaneously, we perform a single-point aerodynamic shape optimization with wing and tail shape variables. The total number of 


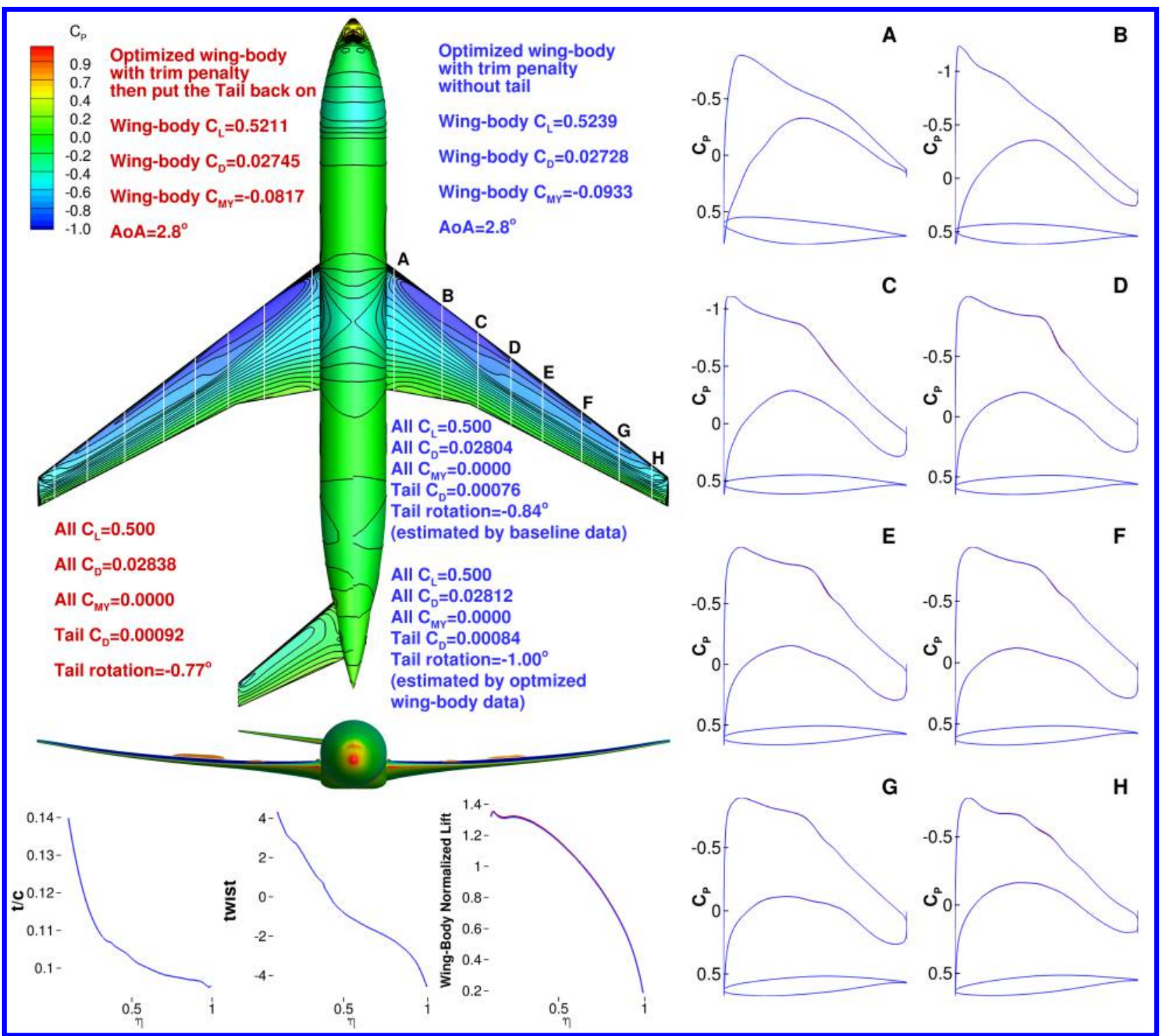

Figure 14. Comparison between the optimized wing-body with trim penalty and the same configuration with a tail added back on and trimmed.

shape design variables is $816+144=960$.

Figure 18 shows the comparison between the configuration optimized with respect to wing and tail shape simultaneously, and the configuration optimized with respect to wing shape alone. The optimizer reduced the lift on the wing in the simultaneously optimized one by making the lift on the tail slightly positive. The difference in drag between these two cases is within one drag count, which is a relatively small amount compared with the reduction between the baseline and the wing only optimized configuration.

The moment coefficient value of the simultaneously optimized configuration is $C_{M_{y}}=-0.133$, the absolute value of which is much higher than that of the wing only optimized one $C_{M_{y}}=-0.0782$. Figure 19 shows the comparison between the shape and pressure coefficient distribution on the tail for the simultaneously optimized configuration with the one optimized with respect to wing shape alone. We can see that the optimizer changed the lift on the tail by adding aft-loading on the inboard of the tail, while maintaining similar pressure distribution on the outboard part. 


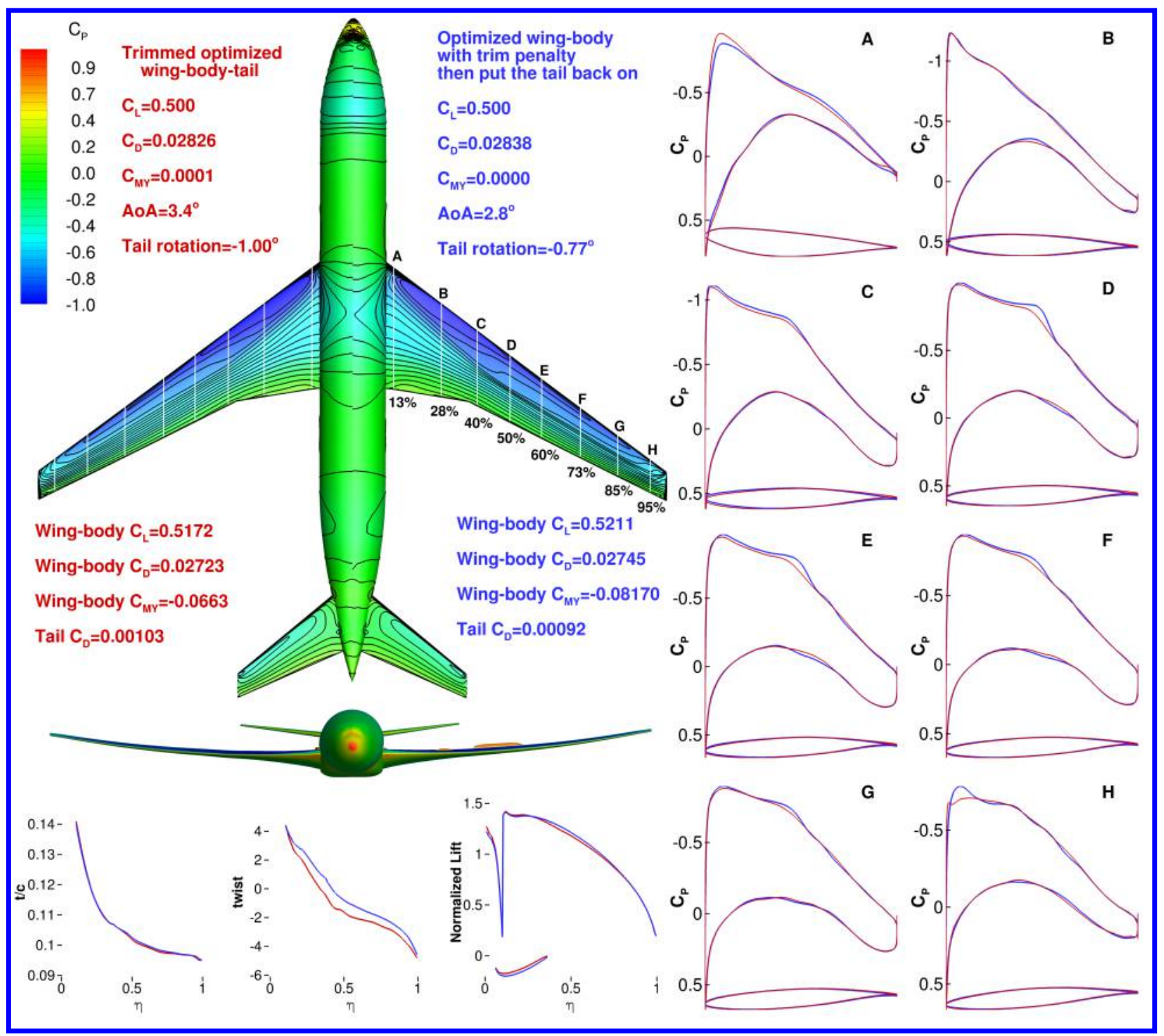

Figure 15. Comparison of the optimized wing-body with trim penalty and tail added back on with the trim-constraint optimized wingbody-tail. 


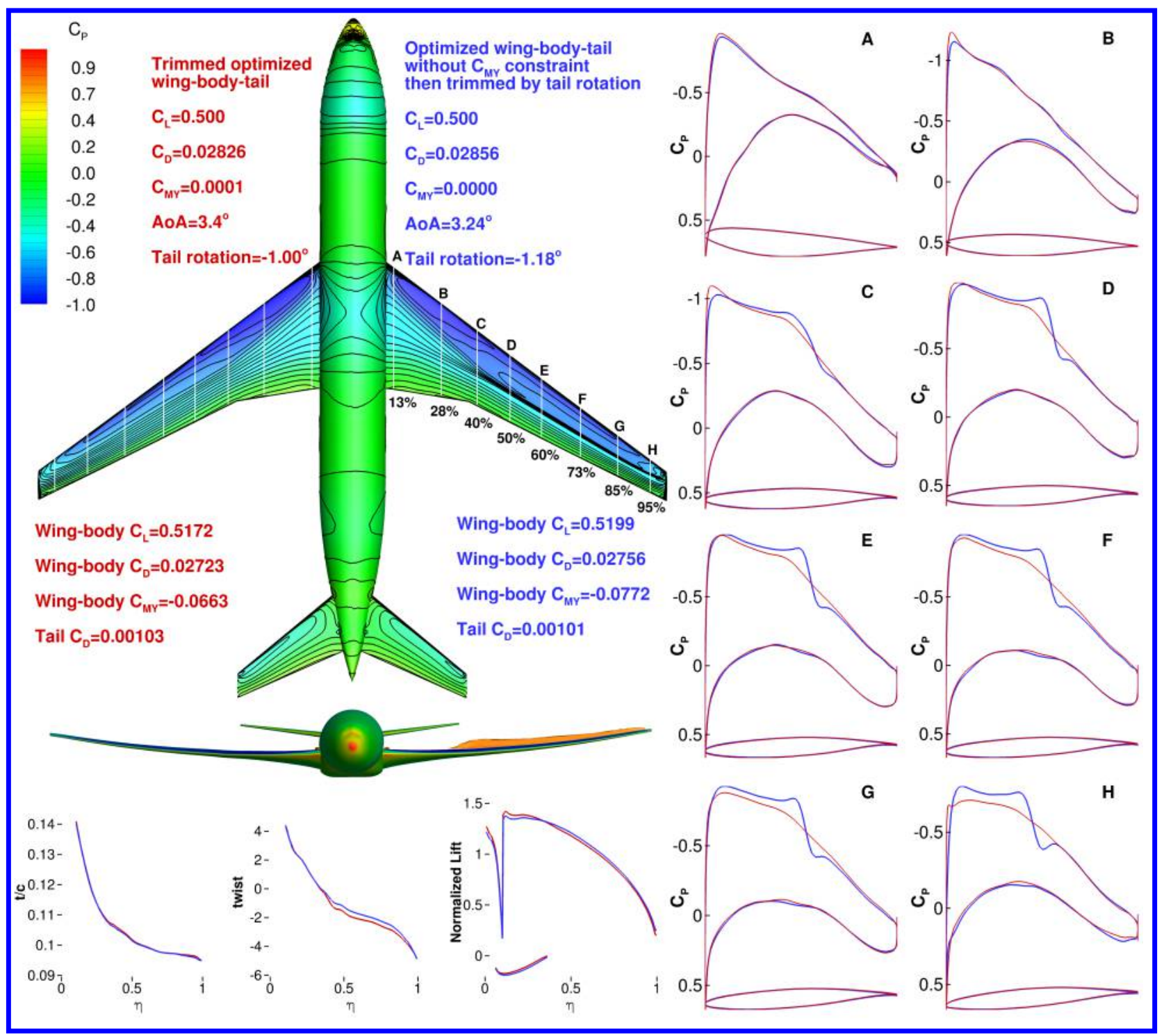

Figure 16. Comparison of the optimized wing-body-tail without trim constraint and trimmed by rotating tail after optimization with the trim-constraint optimized wing-body-tail. 


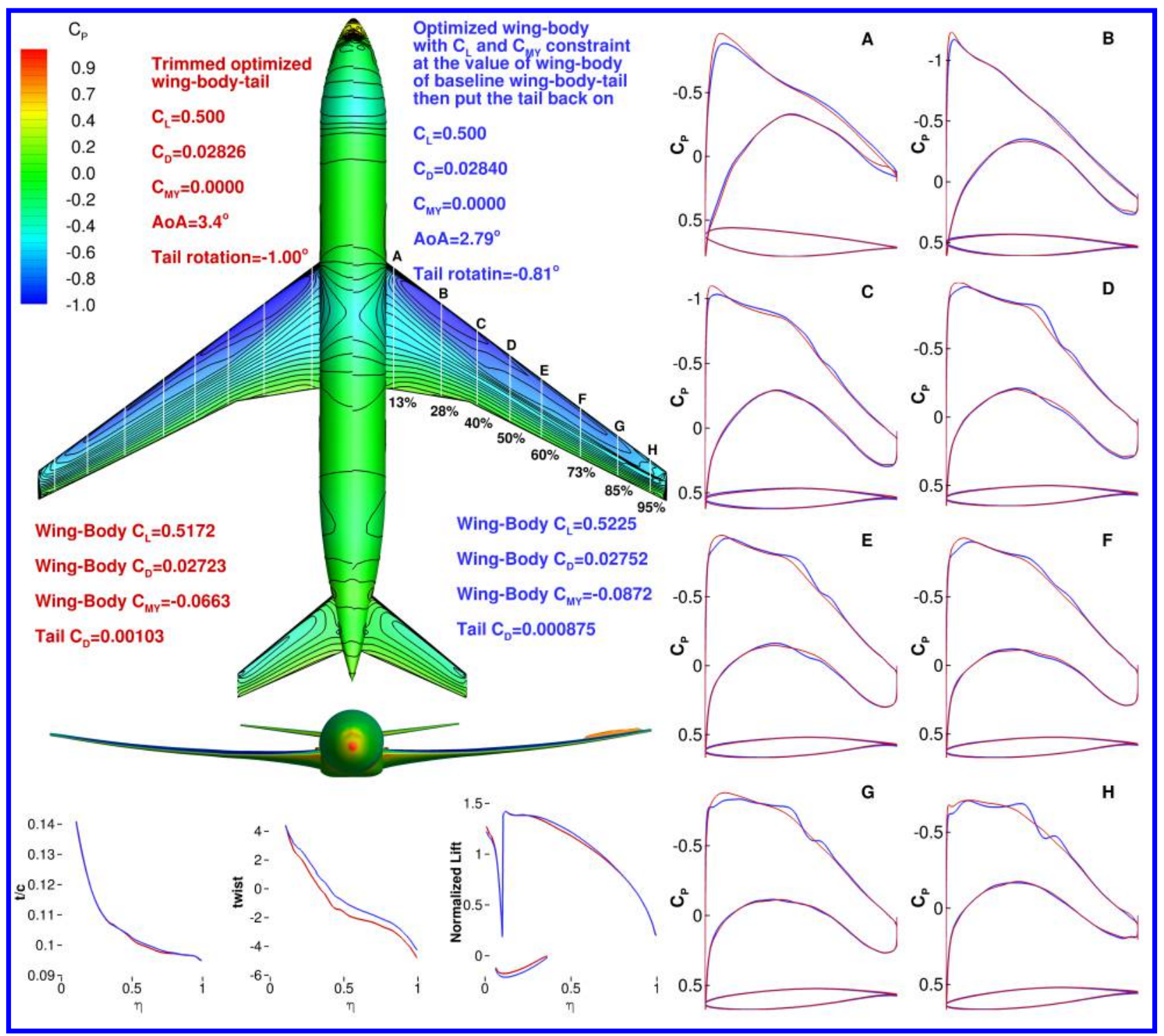

Figure 17. Comparison of the optimized wing-body-tail without trim constraint and trimmed just by tail rotation after optimization to the trimmed optimized wing-body-tail. 


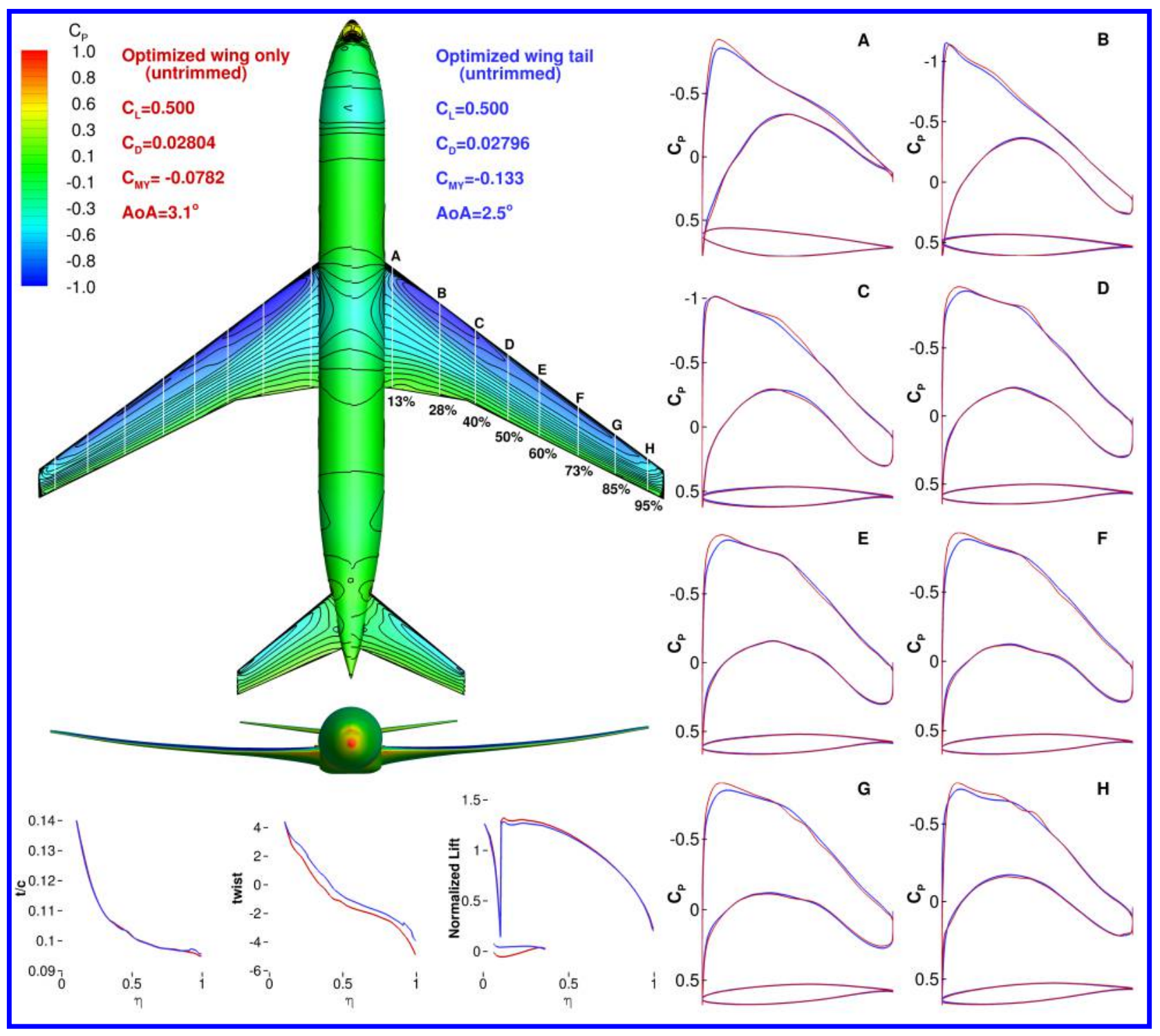

Figure 18. The untrimmed wing and tail simultaneously optimized one.

\section{Simultaneous Aerodynamic Shape Optimization of Wing and Tail}

A final design optimization case was investigated where we added horizontal tail shape variables to simultaneously optimized the wing shape and the tail shape, hoping to further improve the aerodynamic efficiency of the full trimmed configuration.

Figure 20 shows the comparison between the trim-constrained wing and tail shape optimization with the trimconstrained wing optimization. In this case, the drag reduction is even less than the previous one: approximately 0.3 drag counts. Figure 21 shows that the optimizer has not changed the pressure distribution on the tail significantly, so that the lift distribution on the tail is only slightly different between the two. The $-\partial C_{M} / \partial C_{L}$ of this optimized configuration is $35.74 \%$, very closed to that value of the one without the shape design variables on the tail, also higher than that value of the baseline which is $30 \%$.

We make a final comparison between the results from the previous design optimization cases in Table 3 . The difference between these optimization cases are the design variables and the moment constraint. In the table the first 


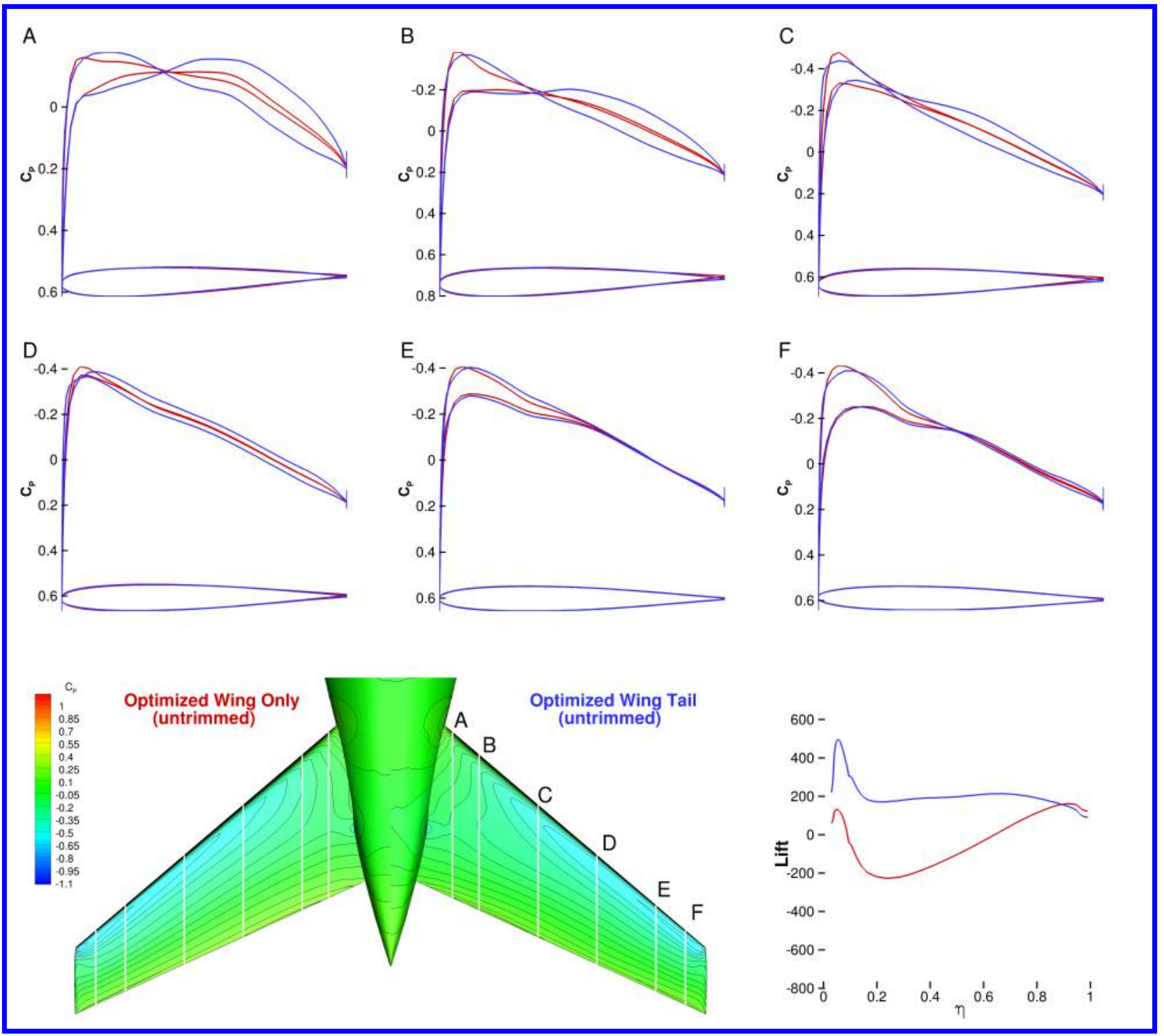

Figure 19. The tail of the untrimmed wing and tail simultaneously optimized one.

is the baseline and the second is the trimmed baseline with rotated tail. The third is with only shape design variables on the wing without a moment constraint, the fourth is the optimization with respect to both shape design variables on the wing and the tail without a moment constraint, the fifth is the optimization with respect to shape design variables on the wing and the tail rotation variable with the trim constraint, the sixth one is the optimization of wing-body with respect to shape design variables on the wing together with a constraint from estimated trim penalty on the tail, the seventh is the optimization of wing-body with respect to shape design variables on the wing together with $C_{L}$ and $C_{M_{y}}$ constraint from the value of wing-body component within the trimmed baseline wing-body-tail configuration, and the last one is the optimization with respect to both shape design variables on the wing and the tail,together with the tail rotation variable for trimming. This comparison shows that using trimming constraint by real tail rotation could achieve a lower drag value than the ones with either estimated trim penalty or fixed moment constraint. It also shows that with the trim constraint, adding shape design variables on the tail does not reduce the overall drag significantly: it result in a drag reduction of 0.3 counts. 


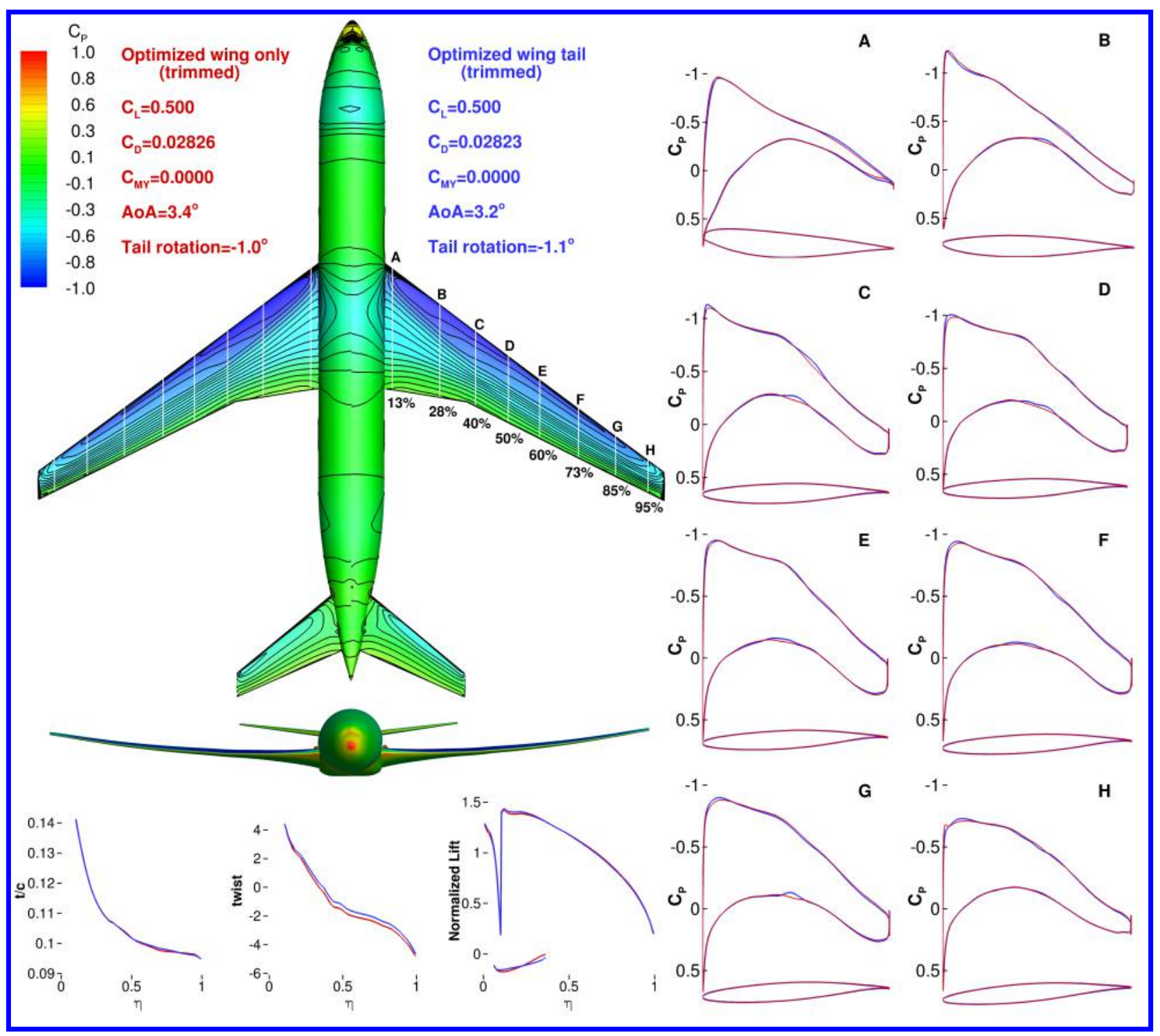

Figure 20. Comparision between wing and tail shape optimization versus wing only optimization.

We also compared the $C_{M_{y}}{ }^{-} C_{L}$ curve and the $C_{D}-C_{L}$ curve of four configurations above, which are the baseline, the baseline with tail rotated to be trimmed at $C_{L}=0.5$, the optimized wing shape with tail rotated to be trimmed at $C_{L}=0.5$ and the optimized wing and tail shape with tail rotated to be trimmed at $C_{L}=0.5$ in Fig 22. It shows that with rotating the tail the curve of the baseline(red curve) has shifted above, and the optimized configuration have maintained nearly same trend and slope within the linear region. Around the design condition the optimized ones exhibit slightly non-linear while the baseline well maintains the linear. Besides, as the $C_{L}$ increases, the $C_{M_{y}}$ of both of the optimized ones turn increasing earlier than the baseline. As for the $C_{D}-C_{L}$ curve, both of the optimized ones have a lower drag than the baseline at around the designed $C_{L}$ and higher $C_{L}$, however sacrificing the performance at lower $C_{L}$ from 0.2 to 0.45 approximately. 


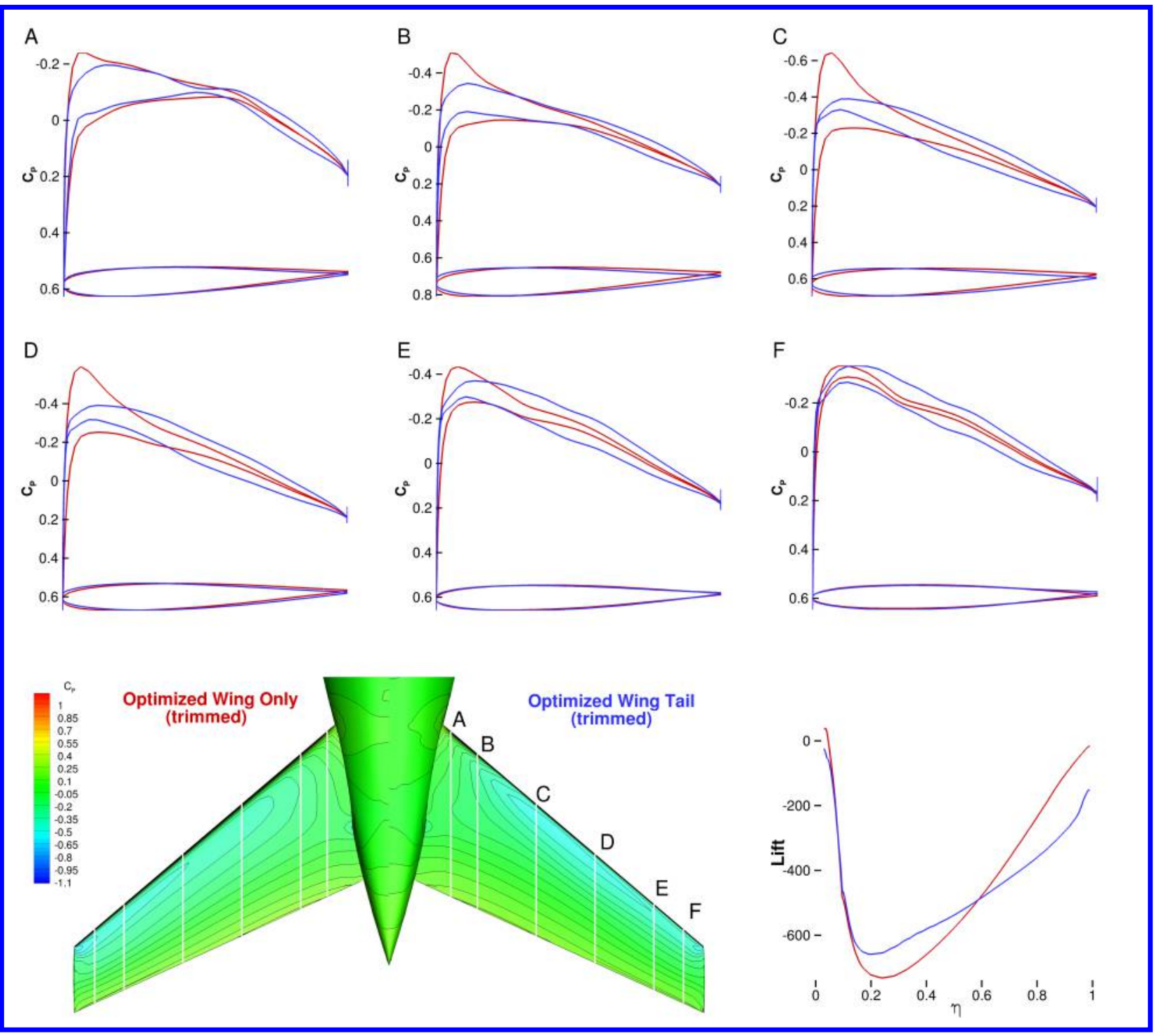

Figure 21. Tail detail comparison between wing and tail shape optimization versus wing only optimization.

\section{Conclusions}

In this paper, we set out to find the value of including trim during the aerodynamic shape optimization of a conventional aircraft configuration. To this end, we performed a series of optimizations of the CRM wing-body-tail and wing-body configurations. We minimized the drag coefficient subject to lift, pitching moment, and geometric constraints. The optimizations were performed on two mesh levels with 746,000 and 5.97 million cells, using 816 shape design variables on the wing and 144 shape design variables on the tail, together with angle of attack and horizontal tail rotation angle.

For optimizations that included trim constraints, the one with tail simultaneously rotated within the optimization process achieved the lowest overall drag. The single-point trim-constrained optimization achieved 12.1 drag counts less than the trimmed baseline, which amounts to $4.1 \%$ of the total drag of the trimmed baseline.

The aerodynamic shape optimization of the wing-body without the tail was performed by implementing a surrogate model of the trim drag penalty to account for the trade between the wing performance and the trim penalty on the tail. 


\begin{tabular}{|c|c|c|c|c|c|}
\hline Case & $C_{L}$ & $C_{D}$ & $C_{M_{y}}$ & Tail rotation & AoA \\
\hline Baseline & 0.5 & 0.02907 & -0.0410 & 0.0 & 2.41 \\
\hline Baseline trimmed & 0.5 & 0.02947 & 0.0 & -0.6 & 2.50 \\
\hline Optimized wing shape & 0.5 & 0.02804 & -0.0780 & 0.0 & 3.09 \\
\hline Optimized wing shape and tail Shape & 0.5 & 0.02796 & -0.1326 & 0.0 & 2.53 \\
\hline Trimmed optimized wing shape & 0.5 & 0.02826 & 0.0 & -1.0 & 3.42 \\
\hline $\begin{array}{l}\text { Optimized wing-body } \\
\text { with estimated trim penalty, } \\
\text { then trimmed with tail back on }\end{array}$ & 0.5 & 0.02838 & 0.0 & -0.8 & 2.80 \\
\hline $\begin{array}{l}\text { Optimized wing-body } \\
\text { with } C_{L} \text { and } C_{M_{y}} \text { constraints from baseline, } \\
\text { then trimmed with tail back on }\end{array}$ & 0.5 & 0.02840 & 0.0 & -0.8 & 2.80 \\
\hline Trimmed optimized wing shape and tail shape & 0.5 & 0.02823 & 0.0 & -1.1 & 3.17 \\
\hline
\end{tabular}

Table 3. Comparison of the results of all cases.

When adding the tail back on the optimized wing-body with this trim penalty, the configuration had 1.2 drag counts more than the trim-constrained optimized wing-body-tail. Thus if such drag coefficient differences are important to the designer, the trim-constrained optimization with the horizontal tail rotation is preferred. Otherwise, a designer can use the trim drag surrogate model to get results that are approximate enough.

When adding the shape design variables for the tail, the trim-constrained optimization reduces the drag by only 0.3 counts. Overall, these results show that the baseline CRM configuration is already well designed from the trim point of view, with a reasonable wing moment coefficient. The value of performing aerodynamic shape optimization considering trim would increase further if we considered multiple center of gravity positions, or if we started from a baseline wing with a larger pitch down moment.

\section{Acknowledgments}

This work used the Extreme Science and Engineering Discovery Environment (XSEDE) [37], which is supported by National Science Foundation grant number ACI-1053575. Part of the work were performed on the Flux HPC cluster at the University of Michigan Center of Advanced Computing. 


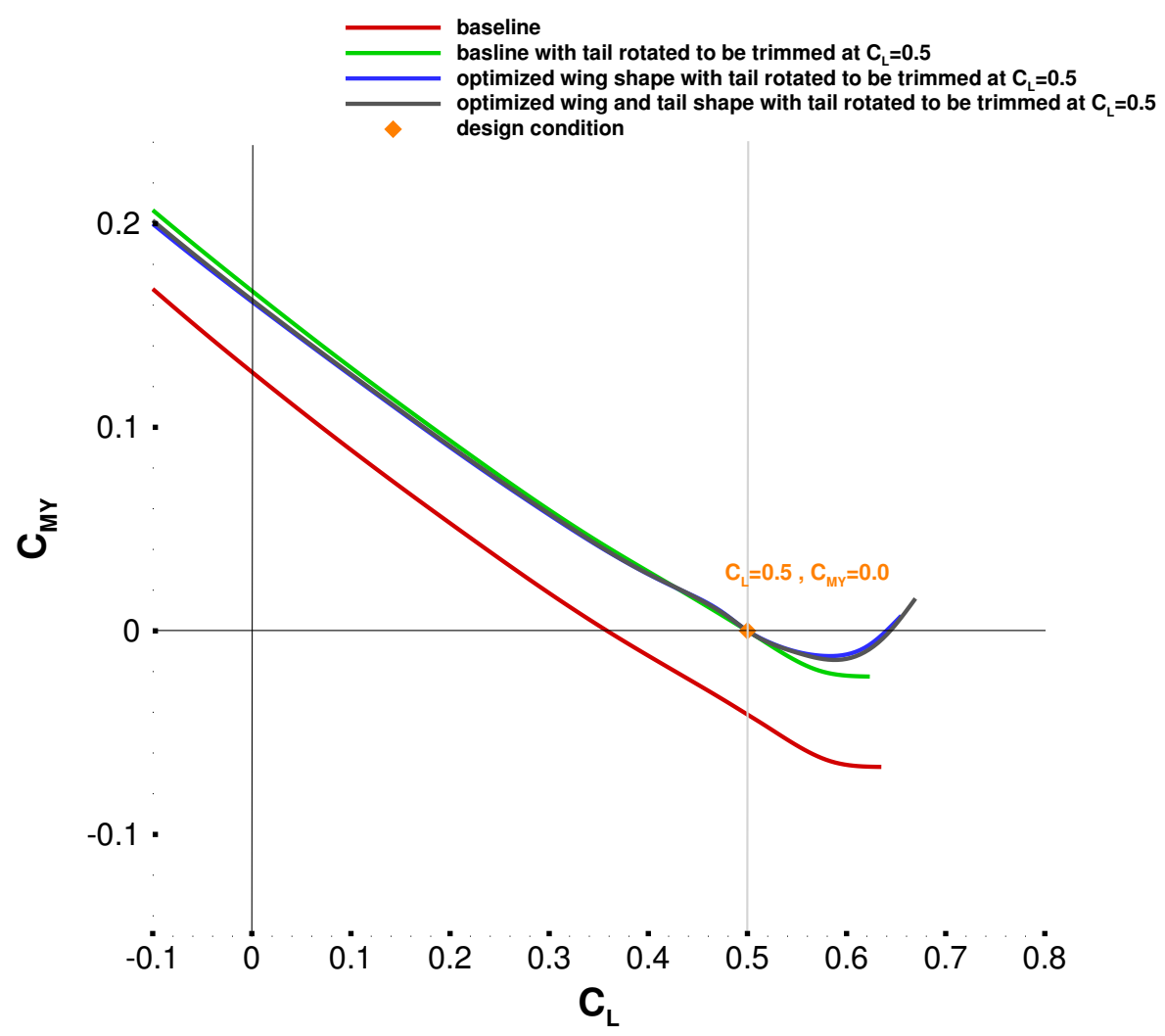

(a) $C_{M_{y}}-C_{L}$

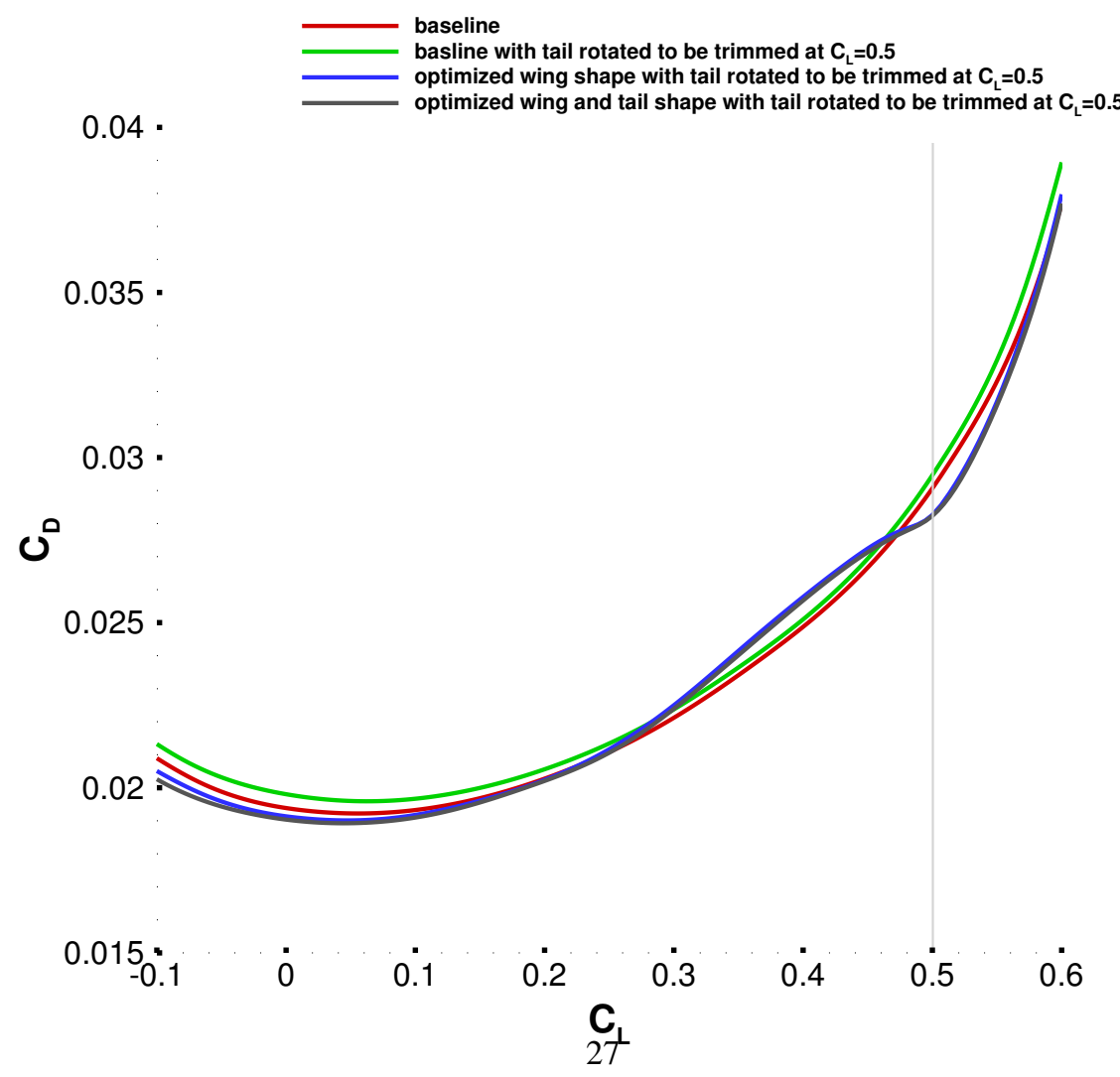

(b) $C_{D}-C_{L}$

Figure 22. Comparison of the $C_{M_{y}}-C_{L}$ curve and $C_{D}-C_{L}$ curve. 


\section{References}

- [1] Jameson, A., “Aerodynamic Design via Control Theory," Journal of Scientific Computing, Vol. 3, No. 3, 1988, pp. $233-260$. doi:10.1007/BF01061285.

[2] Peter, J. E. V. and Dwight, R. P., "Numerical Sensitivity Analysis for Aerodynamic Optimization: A Survey of Approaches," Computers and Fluids, Vol. 39, 2010, pp. 373-391. doi:10.1016/j.compfluid.2009.09.013.

[3] Lyu, Z., Kenway, G. K. W., and Martins, J. R. R. A., "Aerodynamic Shape Optimization Investigations of the Common Research Model Wing Benchmark," AIAA Journal, 2014, (In press).

- [4] Lyu, Z. and Martins, J. R. R. A., "Aerodynamic Design Optimization Studies of a Blended-Wing-Body Aircraft," Journal of Aircraft, 2014. doi:10.2514/1.C032491.

-[5] I.Kroo, “Tail Sizing for Fuel Efficient Transports," AIAA Aircraft Design, Systems and Technology Meeting, October 1983. doi:10.2514/6.1983-2476.

-[6] Lyu, Z., Kenway, G., Paige, C., and Martins, J. R. R. A., "Automatic Differentiation Adjoint of the Reynolds-Averaged Navier-Stokes Equations with a Turbulence Model," 43rd AIAA Fluid Dynamics Conference and Exhibit, June 2013.

-[7] Vassberg, J., Dehaan, M., Rivers, M., and Wahls, R., "Development of a Common Research Model for Applied CFD Validation Studies," 26th AIAA Applied Aerodynamics Conference, American Institute of Aeronautics and Astronautics, August 2008. doi:10.2514/6.2008-6919.

-[8] Reuther, J. J., Jameson, A., Alonso, J. J., Rimlinger, M. J., and Saunders, D., "Constrained Multipoint Aerodynamic Shape Optimization Using an Adjoint Formulation and Parallel Computers, Part 1," Journal of Aircraft, Vol. 36, No. 1, 1999, pp. 5160. doi:10.2514/2.2413.

-[9] Reuther, J. J., Jameson, A., Alonso, J. J., Rimlinger, M. J., and Saunders, D., "Constrained Multipoint Aerodynamic Shape Optimization Using an Adjoint Formulation and Parallel Computers, Part 2," Journal of Aircraft, Vol. 36, No. 1, 1999, pp. 6174. doi:10.2514/2.2414.

[10] Nielsen, E. J. and Anderson, W. K., "Aerodynamic Design Optimization on Unstructured Meshes Using the Navier-Stokes Equations," AIAA journal, Vol. 37, No. 11, 1999, pp. 1411-1419. doi:10.2514/2.640.

-[11] Nemec, M., Zingg, D. W., and Pulliam, T. H., "Multipoint and Multi-Objective Aerodynamic Shape Optimization," AIAA Journal, Vol. 42, No. 6, 2004, pp. 1057-1065. doi:10.2514/1.10415.

- [12] Bueno-Orovio, A., Castro, C., Palacios, F., and Zuazua, E., "Continuous Adjoint Approach for the Spalart-Allmaras Model in Aerodynamic Optimization,” AIAA Journal, Vol. 50, No. 3, 2012, pp. 631-646. doi:10.2514/1.J051307.

-[13] Hicken, J. E. and Zingg, D. W., "Induced-Drag Minimization of Nonplanar Geometries Based on the Euler Equations," AIAA journal, Vol. 48, No. 11, 2010, pp. 2564-2575. doi:10.2514/1.J050379.

[14] Vassberg, J. and Jameson, A., "Influence of Shape Parameterization on Aerodynamic Shape Optimization," Tech. rep., Von Karman Institute, Brussels, Belgium, April 2014.

[15] Telidetzki, K., Osusky, L., and Zingg, D. W., "Application of Jetstream to a Suite of Aerodynamic Shape Optimization Problems," 52nd Aerospace Sciences Meeting, Feb 2014. doi:10.2514/6.2014-0571.

- [16] Carrier, G., Destarac, D., Dumont, A., Meheut, M., Din, I. S. E., Peter, J., Khelil, S. B., Brezillon, J., and Pestana, M., "Gradient-Based Aerodynamic Optimization with the elsA Software," 52nd Aerospace Sciences Meeting, Feb 2014. doi:10.2514/6.2014-0568.

[17] Liem, R., Kenway, G. K. W., and Martins, J. R. R. A., "Multimission Aircraft Fuel Burn Minimization via Multipoint Aerostructural Optimization,” AIAA Journal, 2014. doi:10.2514/1.J052940, (In press).

[18] Kenway, G. K. W. and Martins, J. R. R. A., "Multipoint High-fidelity Aerostructural Optimization of a Transport Aircraft Configuration," Journal of Aircraft, Vol. 51, No. 1, 2014, pp. 144-160. doi:10.2514/1.C032150.

-[19] Mader, C. A. and Martins, J. R. R. A., "Stability-Constrained Aerodynamic Shape Optimization of Flying Wings," Journal of Aircraft, Vol. 50, No. 5, September 2013, pp. 1431-1449. doi:10.2514/1.C031956.

[20] Reist, T. A. and Zingg, D. W., "Aerodynamically Optimal Regional Aircraft Concepts: Conventional and Blended-Wing-Body Designs," 52nd Aerospace Sciences Meeting, Jan 2014. doi:10.2514/6.2014-0905.

[21] E.V.Laitone, "Ideal Tail Load for Minimum Aircraft Drag," Journal of Aircraft, March 2006, pp. $190-192$. doi: $10.2514 / 3.58339$.

[22] W.F.Phillips, A.B.Hansen, W., "Effects of Tail Dihedral on Static Stability," Journal of Aircraft, November 2006, pp. 18291837. doi:10.2514/1.20683. 
[23] Kenway, G. K. W., A Scalable, Parallel Approach for Multi-Point, High-Fidelity Aerostructural Optimization of Aircraft Configurations, Ph.D. thesis, University of Toronto, 2013.

-[24] Kenway, G. K. W., Kennedy, G. J., and Martins, J. R. R. A., "Scalable Parallel Approach for High-Fidelity SteadyState Aeroelastic Analysis and Adjoint Derivative Computations," AIAA Journal, Vol. 52, No. 5, 2014, pp. $935-951$. doi:10.2514/1.J052255.

[25] Kenway, G. K. W., Kennedy, G. J., and Martins, J. R. R. A., "Aerostructural optimization of the Common Research Model configuration," 15th AIAA/ISSMO Multidisciplinary Analysis and Optimization Conference, Atlanta, GA, June 2014, AIAA 2014-3274.

[26] Kenway, G. K., Kennedy, G. J., and Martins, J. R. R. A., "A CAD-free Approach to High-Fidelity Aerostructural Optimization," Proceedings of the 13th AIAA/ISSMO Multidisciplinary Analysis Optimization Conference, Fort Worth, TX, 2010. doi:10.2514/6.2010-9231.

[27] van der Weide, E., Kalitzin, G., Schluter, J., and Alonso, J., "Unsteady Turbomachinery Computations Using Massively Parallel Platforms," 44th AIAA Aerospace Sciences Meeting and Exhibit, 2006. doi:10.2514/6.2006-421.

[28] Jameson, A., Schmidt, W., and Turkel, E., "Numerical Solution of the Euler equations by Finite Volume Methods Using Runge Kutta Time Stepping Schemes,” 14th AIAA, Fluid and Plasma Dynamics Conference, 1981.

-[29] Saad, Y. and Schultz, M. H., "GMRES: A Generalized Minimal Residual Algorithm for Solving Nonsymmetric Linear Systems," SIAM Journal on Scientific and Statistical Computing, Vol. 7, No. 3, 1986, pp. 856-869. doi:10.1137/0907058.

-[30] Balay, S., Gropp, W. D., McInnes, L. C., and Smith, B. F., "Efficient Management of Parallelism in Object Oriented Numerical Software Libraries," Modern Software Tools in Scientific Computing, edited by E. Arge, A. M. Bruaset, and H. P. Langtangen, Birkhäuser Press, 1997, pp. 163-202. doi:10.1007/978-1-4612-1986-6_8.

[31] Balay, S., Brown, J., , Buschelman, K., Eijkhout, V., Gropp, W. D., Kaushik, D., Knepley, M. G., McInnes, L. C., Smith, B. F., and Zhang, H., "PETSc Users Manual," Tech. Rep. ANL-95/11 - Revision 3.4, Argonne National Laboratory, 2013.

[32] Balay, S., Brown, J., Buschelman, K., Gropp, W. D., Kaushik, D., Knepley, M. G., McInnes, L. C., Smith, B. F., and Zhang, H., "PETSc Web page," 2013, http://www.mcs.anl.gov/petsc.

[33] Gill, P. E., Murray, W., and Saunders, M. A., "SNOPT: An SQP Algorithm for Large-Scale Constrained Optimization,” SIAM journal on optimization, Vol. 12, No. 4, 2002, pp. 979-1006. doi:10.1137/S1052623499350013.

- [34] Perez, R. E., Jansen, P. W., and Martins, J. R. R. A., "pyOpt: A Python-Based Object-Oriented Framework for Nonlinear Constrained Optimization," Structures and Multidisciplinary Optimization, Vol. 45, No. 1, 2012, pp. 101-118. doi:10.1007/s00158-011-0666-3.

[35] Lyu, Z., Kenway, G. K. W., and Martins, J. R. R. A., "RANS-based Aerodynamic Shape Optimization Investigations of the Common Research Model Wing," AIAA Science and Technology Forum and Exposition (SciTech), National Harbor, MD, January 2014.

[36] Roache, P. J., "Verification of codes and calculations," AIAA journal, 1998.

-[37] Towns, J., Cockerill, T., Dahan, M., Foster, I., Gaither, K., Grimshaw, A., Hazlewood, V., Lathrop, S., Lifka, D., Peterson, G. D., Roskies, R., Scott, J. R., and Wilkens-Diehr, N., "XSEDE: Accelerating Scientific Discovery," Computing in Science and Engineering, 2014. doi:10.1109/MCSE.2014.80. 
This article has been cited by:

1. David Koo, David W. Zingg. Investigation into Aerodynamic Shape Optimization of Planar and Nonplanar Wings. AIAA Journal, ahead of print1-14. [Abstract] [Full Text] [PDF] [PDF Plus] 